\title{
Comparison of Computational and Experimental Microphone Array Results for an 18\%-Scale Aircraft Model
}

\author{
David P. Lockard, ${ }^{*}$ William M. Humphreys, ${ }^{\dagger}$ Mehdi R. Khorrami $^{\ddagger}$ \\ NASA Langley Research Center, Hampton, VA 23681 USA \\ Ehab Fares, ${ }^{\S}$ Damiano Casalino $₫$ \\ Exa GmbH, Curiestrasse 4, D-70563 Stuttgart, Germany \\ and \\ Patricio A. Ravettall \\ AVEC, Inc, Blacksburg, VA 24060
}

\begin{abstract}
An 18\%-scale, semi-span model is used as a platform for examining the efficacy of microphone array processing using synthetic data from numerical simulations. Two hybrid RANS/LES codes coupled with Ffowcs Williams-Hawkings solvers are used to calculate 97 microphone signals at the locations of an array employed in the NASA LaRC $14 \times 22$ tunnel. Conventional, DAMAS, and CLEAN-SC array processing is applied in an identical fashion to the experimental and computational results for three different configurations involving deploying and retracting the main landing gear and a part span flap. Despite the short time records of the numerical signals, the beamform maps are able to isolate the noise sources, and the appearance of the DAMAS synthetic array maps is generally better than those from the experimental data. The experimental CLEAN-SC maps are similar in quality to those from the simulations indicating that CLEAN-SC may have less sensitivity to background noise. The spectrum obtained from DAMAS processing of synthetic array data is nearly identical to the spectrum of the center microphone of the array, indicating that for this problem array processing of synthetic data does not improve spectral comparisons with experiment. However, the beamform maps do provide an additional means of comparison that can reveal differences that cannot be ascertained from spectra alone.
\end{abstract}

\section{Introduction}

The use of numerical simulations in the prediction of airframe noise sources is becoming relatively routine. Indeed, simulations have even been used as an initial screening tool to down select noise reduction devices for more detailed experimental study. ${ }^{1}$ When care is taken to ensure that the simulations are capturing all of the salient flow features, they can be an important and complementary tool to experimentation. In aeroacoustic testing, the phased array has become a standard measurement technique because it can exclude unwanted extraneous noise sources, but also provides guidance on noise source locations. Conventional beamforming phased-array processing involves assuming an acoustic source basis function (such as a monopole in a uniform flow) and placing one of these sources at every point in a mesh surrounding a region where sound sources are expected. A source strength amplitude for each grid point is determined by how well the signals across the microphone array are consistent with the assumed basis function. Using the distances between the grid point and the microphones, each signal is adjusted in amplitude and time (or phase). They can then be combined with the portion of the signal consistent with the assumed basis adding up constructively, whereas the inconsistent portion combines destructively. However, this assumes that the sources are uncorrelated and their directivity is consistent with the basis function, which is typically a monopole. Furthermore, even when the source coincides exactly with the assumed basis, the array response is dependent on the particular arrangement of the microphones relative to the sources. Deconvolution methods have been developed to account for the array response that can provide spectra equivalent to what would be obtained by a single microphone, but all of these algorithms require certain assumptions and some can be computationally expensive. Nonetheless, microphone arrays have provided valuable information about noise sources when the elevated background noise would render single microphone measurements useless. In particular, the contour maps of source strength provide information about the location of sources that was not available previously.

\footnotetext{
* Aerospace Engineer, Computational AeroSciences Branch, Senior Member, AIAA

$\dagger$ Assistant Branch Head, Advanced Measurements and Data Systems Branch, Associate Fellow AIAA

$\ddagger$ Aerospace Engineer, Computational AeroSciences Branch, Associate Fellow AIAA

$\S$ Technical Director, Aerospace Applications, Senior Member AIAA

I Technical Director, Aerospace Aeroacoustics, Senior Member AIAA

${ }$ Chief Research Engineer, Senior Member AIAA
} 
However, even when array data identifies a strong acoustic source, it provides no insight into the flow features giving rise to the source. The connection to underlying flow features is often what is most useful in developing the understanding necessary for the design of noise reduction devices. Although additional experimental studies, such as optical measurements, can help make these connections, simulation data provides another means of interrogating the flow.

A methodology commonly used to make aeroacoustic predictions using computational fluid dynamics (CFD) involves coupling the near-field solution from the CFD to an acoustic analogy such as the Ffowcs Williams and Hawking's equation $^{2}(\mathrm{FW}-\mathrm{H})$. These predictions are typically computed at the center of the array and compared with the array output. However, with a minimal increase in computational cost, the predictions can be made at all microphone locations in the array, and the signals processed in the same manner as experimental data. Synthetic array data coming from analytic solutions has been used for years in the design of microphone arrays, and recently numerical acoustic propagation codes have been used to test out advanced beamforming algorithms for rotating machinery. ${ }^{3}$ The combination of CFD and FW$\mathrm{H}$ has been used to compute synthetic array data for wind turbines ${ }^{4}$ and supersonic jets. ${ }^{5}$ In the latter investigation, the beamforming results were able to identify numerical issues giving rise to nonphysical sound sources. Although synthetic data from CFD does not suffer from many of the extraneous noise sources typically found in an experiment (such as wind tunnel fan noise and noise generation or reflection from the facility), numerical artifacts can just as easily contaminate acoustic results. Nonetheless, these effects are typically identifiable and correctable when they exist. However, the time record lengths that can be obtained from CFD are orders of magnitude shorter than what is typically available from an experiment. If the relatively clean nature of the numerical signals allows relatively short record lengths to be used in the beamforming process, then a more direct comparison with the experimental result can be obtained. Furthermore, additional insight into the noise sources may be gained by querying the CFD.

\section{Approach}

The current study involves an 18\%-scale, high-fidelity, semi-span Gulfstream aircraft model that was tested in the NASA Langley Research Center (LaRC) 14- by 22-foot subsonic tunnel. ${ }^{1}$ The model includes a part-span flap that was set at three angles and a removable landing gear. Data was collected at three Mach numbers and three angles of attack, but the present work only examines the Mach $0.2,3^{\circ}$ angle of attack case with three configurations: landing gear deployed and retracted with the flap set at $39^{\circ}$ and landing gear deployed with the flap set at $0^{\circ}$. Extensive steady and unsteady surface pressure data ${ }^{6}$ as well as particle image velocimetry results ${ }^{7}$ have been compared with corresponding information from high-fidelity unsteady, hybrid RANS/LES computations using both Exa Corporation's PowerFLOW ${ }^{\circledR}$ code $^{8}$ and the FUN3D code ${ }^{9}$ developed at NASA LaRC. Favorable aeroacoustic comparisons have been made between the numerical simulations and experimental array output. The new component of this work involves using the existing simulations to predict the pressure signals at all microphone locations and processing these signals with several different beamforming algorithms. During the experimental testing, the quick look capability in the AVEC, Inc. beamforming suite $^{10}$ was used to assess the quality of the acquired data. This included standard beamforming processing and some more detailed analysis using the CLEAN-SC ${ }^{11}$ deconvolution algorithm. The NASA UDAMAS code was used after the test to perform standard beamforming and DAMAS ${ }^{12}$ processing. In addition, sample results from the recently developed Exa beamforming code that is based on the CLEAN-SC ${ }^{11}$ approach is included in the comparisons.

\section{Results}

For all of the investigated configurations, the flow speed is Mach 0.2 with the aircraft at an angle of attack of $3^{\circ}$. The mean aerodynamic chord of the model is 30.8 in $(0.782 \mathrm{~m})$, which corresponds to a Reynolds number of $3.40 \times 10^{6}$. A picture of the model is shown in Fig. 1, and an image showing the array in the background of the model is shown in Fig. 2. The array is positioned in the $90^{\circ}$ position, geometrically directly beneath the aircraft. However, because of the shear layer in the open-jet tunnel, the actual overhead position (emission angle) is closer to 83.1 ${ }^{\circ}$ The 97-microphone array is 210 inches $(5.33 \mathrm{~m}$ ) from the tunnel centerline, with an outer diameter (microphone to microphone) of 78.6 inches $(2.0 \mathrm{~m})$. An array shading algorithm was employed to exclude certain microphones based on the frequency so that sources appear similar in size across the frequency range and to reduce the distances between the included microphones as the frequency increases, which helps to minimize the detrimental effects of decorrelation. Detailed information about the array can be found in Ref. 13.

Both FUN3D 9 and Exa PowerFLOW ${ }^{\circledR 8}$ numerical simulations have been used to calculate the pressure time histories at all 97 microphone locations. These signals are in turn processed in an identical fashion to the experimental data, as detailed in Ref. 1. An array location of $94.2^{\circ}$ is used for the CFD because the computations were performed in free-air, 
without the shear layer. The absence of the shear layer results in the acoustic rays convecting further downstream than those in the experiments, so $94.2^{\circ}$ in the simulations approximates the $90^{\circ}$ position in the experiment.

\section{III.A. Landing Gear Deployed, Flaps at 39}

The baseline landing configuration will be examined first. The duration of the FUN3D signal is 0.137 seconds with a sampling rate of $200 \mathrm{kHz}$ (matching the experiment). This results in seven averages (with $50 \%$ overlap of the data) in the computation of the fast Fourier transforms and a bin width of $24.4 \mathrm{~Hz}$. The duration of the Exa PowerFLOW ${ }^{\circledR}$ signal is 0.269 seconds with a sampling rate of $203 \mathrm{kHz}$ and 15 averages. However, the data was processed slightly differently with the Exa beamforming code where 6 averages (no overlapping) of discrete Fourier transforms results in a bin width of $22.3 \mathrm{~Hz}$. The signals were sampled for 30 seconds in the experiment, resulting in 244 averages. The array processing in the UDAMAS code is performed by averaging 7 adjacent bands from the underlying Fourier transforms resulting in a bin width of $170.8 \mathrm{~Hz}$. These are then combined into 1/12th octaves to create contour maps of source strength. In the AVEC code, the array processing can be performed directly in the 1/12th octave bands. In the Exa beamformer, the beamforming is performed in the narrow $22.4 \mathrm{~Hz}$ bands, then those results combined to form the 1/12th octave image maps. In the process of trying to understand some of the subtle differences observed in the image maps produced by the different codes, we discovered these issues related to the processing. We did run the AVEC code with the same parameters as the Exa beamformer for one case, and the results are more similar. However, the AVEC and Exa CLEAN$\mathrm{SC}$ beamform contour maps presented in this paper involve the different processing methods already discussed, and they can be used to assess how these often subtle changes can manifest themselves in the results. An additional parameter in the processing involves array shading where microphones are weighted based on their location and the frequency. Originally, some codes used the array shading discussed in Ref. 13, but others used uniform weighting. Using a consistent shading algorthim improved the comparisons, so all of the results presented in the paper use shading. A more thorough investigation of all the processing possibilities is beyond the scope of this paper, but is one of the subjects of the AIAA Aeroacoustics Technical Committee Discussion Group on Array Analysis Methods for Aeroacoustics. ${ }^{14}$

For consistency, all of the DAMAS ${ }^{12}$ spectral results in this paper are presented with the $170.8 \mathrm{~Hz}$ bin width, but CLEAN-SC spectra from the Exa beamformer are shown with the $22.3 \mathrm{~Hz}$ bin width. For the computational results, additional averaging significantly reduces the oscillations in the spectra. Furthermore, the same code was used to compute the cross-spectral matrices used as input to the UDAMAS and AVEC beamforming codes.

A comparison of the power spectral density versus the model-scale frequency is shown in Fig. 3. The experimental and computational results from integrating the DAMAS ${ }^{12}$ beamform maps are compared with the signals from the center microphone in Fig. 3(a), and the more narrow band CLEAN-SC spectra from the Exa beamformer in Fig. 3(b). As expected, the array processing has a bigger effect on the experimental data, which includes more extraneous noise sources that are excluded by the array processing. In general, the center microphone signal and array output for the simulations are in good agreement across the frequency range for both the DAMAS and CLEAN-SC spectra. Overall, the simulations appear to have captured the broadband nature exhibited by the experiment, but the FUN3D spectra significantly falls below the experimental results for frequencies above $7 \mathrm{kHz}$. In this frequency range, the diffusive nature of the simulations has artificially damped out the acoustic waves. The narrow band spectra in Fig. 3(b) clearly shows three distinct tones in the Exa results. The two smaller tones are associated with the outboard flap, but the prominent tone around $2.5 \mathrm{kHz}$ in the spectra is caused by a small cavity in the front post knee joint of the landing gear. This tone was observed at a slightly lower frequency of $2.2 \mathrm{kHz}$ during an initial set of experimental runs (P0547 in 3(a)), but it was eliminated by taping the cavity face at the joint (P1862). Similar action was taken during the grid development stages for the FUN3D-based simulations. The tone around $15 \mathrm{kHz}$ in the FUN3D result is caused by shedding from a hydraulic line that is part of the brake system and is located between the wheels. This shedding may be artificially intense because the simulation may lack sufficient spatial resolution to adequately represent the higher-frequency content that would act to break up the coherent vortex shedding. In the experiment and PowerFLOW ${ }^{\circledR}$ simulation, the noise from the hydraulic line is likely being hidden by the overall broadband noise from the aircraft. The experimental spectra from P1862 exhibit a hump between 7 and $10 \mathrm{kHz}$ that is caused by a resonance in a cavity in the outboard flap tip. Filling the cavity completely eliminated this hump, and even the slight geometric changes associated with an instrumented version of the tip greatly diminished this feature as seen in the spectrum for P0547. The PowerFLOW ${ }^{\circledR}$ simulation seems to pick up this broad tone, but the frequency range is in the 6 to $8 \mathrm{kHz}$ range, closer to what was observed with the instrumented tip. The actual cavity has a compliant seal that was modeled as solid in the simulations, and details such as this may be responsible for the different frequency ranges for the tone. The phenomenon appears to be absent in the FUN3D simulation, possibly because of insufficient spatial resolution resulting in excessive diffusion beyond $6 \mathrm{kHz}$ observed in the results.

The spectral comparison indicates that the computations are doing a remarkable job at predicting the total noise 
from the semi-span model, but a spectrum does not give any indication of the origin of the noise. However, the location of noise sources can be ascertained from array beamforming source maps. These maps have been generated in 1/12th octaves with standard beamforming and the DAMAS ${ }^{12}$ and CLEAN-SC ${ }^{11}$ deconvolution algorithms. The maps are normalized by the peak value, so the maximum source strength is zero. The contours show sources within $10 \mathrm{~dB}$ of the peak, and the images were generated using identical plotting parameters. However, because each image was normalized by its peak, the absolute levels from adjacent figures may be quite different. The reader is directed to the spectral plot in Fig. 3 to ascertain the relative sources strengths. In general, the maps look very similar between the experiment and two computations. Therefore, only a few select frequencies that highlight notable differences will be presented.

Figures 4 and 5 show the conventional beamforming maps in the 1/12th octave band with a center frequency of 2.8 $\mathrm{kHz}$ computed by the NASA UDAMAS and AVEC codes, respectively. The results are nearly identical, and any of the minor discrepancies are likely a result of slight differences in the shear layer corrections (for the experiment) or roundoff caused by the particular implementations used in the codes. Therefore, only the conventional beamforming maps from the UDAMAS code will be presented at other frequencies. Notice that despite the relatively short time duration of the simulated array signals, the conventional beamforming results appear quite reasonable.

Although the conventional beamform maps clearly indicate that the primary noise source is near the inboard flap edge/landing gear in the experiment and simulations, processing with a deconvolution algorithm takes into account the array response, yielding smaller spot sizes and source strengths that are directly related to the noise produced. Indeed, one can directly sum the squared pressure values from DAMAS image maps to obtain the total noise generated by a region. Figure 6 shows the DAMAS response for $2.8 \mathrm{kHz}$, and Fig. 7 shows the results obtained using CLEAN-SC processing. Overall, the DAMAS results for the simulations look cleaner than for the experiment, with the main spots smaller and fewer random sources. At this frequency, the iterative DAMAS processing is having some difficulty converging (visually) in the specified 250 iterations with the experimental data, but seems to be doing relatively well with the simulation data. Increasing the number of DAMAS iterations did not improve the appearance of the images. The results were obtained with cross-spectral matrix diagonal removal performed for 250 DAMAS Gauss-Seidel passes through the measurement grid. Four different sweep directions were used to minimize residual energy migration through the grid. The CLEAN-SC maps all look similar in terms of spot size. Interestingly, the CLEAN-SC results demphasize the source at the outboard tip. The CLEAN-SC processing is performed with a "resolution" parameter that is defined using the parameter $\lambda$ in Eq. 13 of the paper by Sijtsma. ${ }^{11}$ The codes internally define $\lambda=-\log _{10}(0.5) /\left((\text { resolution } / 2)^{2}\right)$ based on the desired resolution, which is defined as the distance where the level is $3 \mathrm{~dB}$ down from the peak. The resolution is set to 3 inches with a loop gain of 0.2 , which is a good compromise among spot size, visual appearance, and processing speed. The output from the AVEC and Exa beamform codes in Figs. 7(a) and (b) compare favorably at this frequency, with the slight difference likely caused by the array shading employed in the AVEC processing.

One reason why the CLEAN-SC experimental maps look better than the ones from DAMAS is because of the way the methods apply the deconvolution. During each iteration, CLEAN-SC identifies the source that appears to have the maximum strength source in the map and then removes that source along with its sidelobes. When the map has a source with a larger mainlobe than can be accounted for by a single source, several 'smaller' sources appear in CLEAN-SC after the iteration. These sources are evident when the cutoff level of the maps is increased beyond the $10 \mathrm{~dB}$ used in this paper. In contrast, DAMAS gradually adjusts the results in each iteration, which leaves the sources looking distributed when the convergence is poor. Because the DAMAS iterative procedure often fails to converge, one cannot simply run more iterations. The experimental data suffers from extraneous noise sources from the wind tunnel and the actual noise from the model has to propagate through a shear layer of around $1 \mathrm{ft}(0.305 \mathrm{~m})$ thick to reach the array. These factors tend to broaden the sources in the conventional beamform image maps as was observed in speaker test cases performed with and without flow. The deconvolution methods use the conventional beamform maps as a starting point, and the level of contamination can diminish the capability of the methods to identify and correctly quantify the sources of interest. CLEAN-SC visually appears to be doing a much better job for this experimental data because the sources appear more compact. However, using a smaller loop gain would increase the number of iterations required to completely remove the energy for a given source. This would result in a larger number of non-zero values around the initial source, making them seem more distributed, and therefore more similar to what is observed with DAMAS. Although the use of a very small loop gain could have an effect on the results, it would dramatically increase the cost of the calculations.

For the synthetic array data, the spectra from all of the methods match up quite well, and agree with the single microphone data. Assuming that the actual sources on the model are similar between the simulations and experiment, the synthetic array data could be modified by adding sources outside of the region of the maps to see how the beamforming results are degraded. Applying the effects of a thick, unsteady shear layer to the synthetic data would also be desirable, but much more difficult. Although these studies are beyond the scope of the current work, they show the potential utility of the synthetic data to analyze array processing techniques and get a better understanding of how they perform when microphone signals are contaminated in ways often experienced during experiments. 
The good agreement between the simulations and experiment seen at $2.8 \mathrm{kHz}$ is indicative of what is observed at most frequencies. Figures 8-10 show the conventional, DAMAS, and CLEAN-SC beamforming maps at $3.0 \mathrm{kHz}$ where, despite this being the adjacent band to the $2.8 \mathrm{kHz}$ shown previously, now some differences are observed. Here, the outboard flap edge is most prominent in the PowerFLOW ${ }^{\circledR}$ simulation, whereas the inboard flap/gear still dominate in the experiment and FUN3D results. Figure 3 shows that the PowerFLOW ${ }^{\circledR}$ spectra has a small peak at this frequency that is absent from the others. The beamform maps at adjacent frequencies all show that the inboard flap dominates, so it is just at this frequency where there is a discrepancy. Because of the short time record in the simulations, the peakiness in the spectra observed in Fig. 3 is generally attributed to insufficient averaging. However, in this case, the beamform result appears to indicate that the peak at $3 \mathrm{kHz}$ in the PowerFLOW ${ }^{\circledR}$ spectra is associated with some physical phenomena in the simulation. This peak could be caused by the outboard flap edge cavity as $3 \mathrm{kHz}$ is a subharmonic of the broad hump associated with this gap. Capturing the details of the tip vortex flow/cavity interaction is obviously very difficult, so differences such as a shift in frequency should be expected.

The results for $8 \mathrm{kHz}$ are shown in Figs. 11-13. At this frequency, the outboard flap edge cavity resonance is responsible for most of the noise in the experiment, but the simulations also indicate some noise coming from the inboard flap/landing gear area. Interestingly, the DAMAS processing greatly reduces the strength of the inboard source compared with the conventional beamforming results. However, at least for the PowerFLOW ${ }^{\circledR}$ simulation, the CLEANSC processing still indicates a relatively strong source around the gear. Furthermore, some hint of noise from the brackets is evident in the PowerFLOW ${ }^{\circledR}$ CLEAN-SC map. Although not evident at this frequency, presumably because of the strong outboard flap edge source, the brackets also appear as secondary sources in some of the experimental data.

A $20 \mathrm{kHz}$ signal (equivalent to $3.6 \mathrm{kHz}$ full scale) is examined in Figs. 14-16. As the spectra in Fig. 3 indicates, the peak levels for the three maps are significantly different, with the FUN3D result being significantly lower in amplitude than the other two. Although the noise at this frequency is significantly less intense than that at lower frequencies, a full-scale value of $3.6 \mathrm{kHz}$ is within the important range for human hearing, so it is informative to see how the array processing algorithms perform at this higher frequency. Although the conventional beamforming maps all look relatively clean, the main spot size in the experimental map is much larger than for the synthetic data. The contamination effects of extraneous wind-tunnel noise and decorrelation through the shear layers tends to broaden the spot sizes more significantly as the frequency increases, and this also contributes to why DAMAS was unable to completely isolate the sources in the experimental data. At high frequencies, significant randomness is evident in the DAMAS maps. However, the DAMAS maps for the synthetic arrays look quite good, with tight sources around flap edges and the gear. In the CLEAN-SC contours, the experimental result looks better than for DAMAS, but the simulation maps have considerably more random sources. Clearly, there are some differences in the results based on the processing technique, and having both experimental and simulated data sets for a configuration may be useful to isolate the causes of the differences.

\section{III.B. Landing Gear Retracted, Flaps at 39॰}

To demonstrate the effect of a configuration change, the case with the landing gear removed and its cavity closed, but the flaps still at $39^{\circ}$, is now examined. This should isolate the sources at the flap edges and on the wing. The data records from the simulations are very similar to that for the previous case, with the FUN3D data record being $0.137 \mathrm{~s}$ long, and $0.270 \mathrm{~s}$ for PowerFLOW ${ }^{\circledR}$.

The power spectral density comparison in Fig. 17 again shows that the array processing significantly lowers the experimental spectra compared with the single microphone result, but there is little change with the simulations. The agreement between the experiment and simulations is remarkably good up to $6 \mathrm{kHz}$, when FUN3D starts to suffer from excessive diffusion and PowerFLOW ${ }^{\circledR}$ predicts the tonal hump caused by the outboard flap cavity. Without the gear, the hump in the PowerFLOW ${ }^{\circledR}$ spectrum is much more evident and clearly resembles the one in the experimental spectrum, albeit in a slightly lower frequency range.

Beamform maps are presented in Figs. $18-25$ at 2.65, 3, 8, and $20 \mathrm{kHz}$ frequencies, but only the DAMAS and CLEAN-SC results are included for brevity as the conventional beamform maps are similar in the quality of appearance to the gear deployed case. Although the outline of the landing gear appears in the aircraft images, it was not included in the simulations nor deployed in the experiment. At 2.65 (Fig. 18), 3 kHz (Fig. 20) and 20 kHz (Fig. 24), the DAMAS maps for the experiment again exhibit some randomness, whereas the maps from the simulated data are quite clean. The $2.65 \mathrm{kHz}$ maps give some indication of bracket noise, although it is more prominent in the DAMAS results. The brackets are clearly a secondary source compared with the flap edges, but they do make a contribution to the total noise that may be important at some frequencies. The source at the inboard edge of the flap is now slightly downstream of what was observed previously, presumably because the noise from the flap is now isolated without a nearby gear source. Also, the gear wake can interfere with the development of the flap side-edge vortex system, and removal of the gear allows the vortex to develop naturally. 
At $3 \mathrm{kHz}$, the PowerFLOW ${ }^{\circledR}$ result still shows a more intense source at the outboard flap, but this is to be expected as the removal of the gear should not have a significant effect on the flow in that region. The CLEAN-SC maps in Fig. 21 all have very well defined sources, and the experimental map again shows an indication of noise from the brackets.

At $8 \mathrm{kHz}$, both the DAMAS (Fig. 22) and CLEAN-SC (Fig. 23) results indicate that the outboard flap edge is the primary source. The CLEAN-SC map for the experiment has a better defined tip source, and the maps for the simulations exhibit some secondary sources slightly above the inboard tip. The $20 \mathrm{kHz}$ results for DAMAS are shown in Fig. 24. Although no clear source is evident in the experimental map, the maps from the simulations clearly show a source at the outboard flap edge. The CLEAN-SC map for the experiment in Fig. 25(a) shows considerably more secondary sources, and the primary sources appear inboard from the tips, similar to what is observed in the simulation results at the inboard edge.

\section{III.C. Landing Gear Deployed, Flaps at $0^{\circ}$}

The final configuration examined has the flaps at $0^{\circ}$ (the stowed condition) and the landing gear deployed. This case isolates the landing gear sources. Only PowerFLOW ${ }^{\circledR}$ has been used to simulate this case, so no FUN3D results will be shown. The data record is once again $0.270 \mathrm{~s}$ in duration for PowerFLOW ${ }^{\circledR}$.

The spectra are compared in Fig. 26, and the prominent tone at $2.5 \mathrm{kHz}$, from the cavity in the knee of the landing gear, is quite evident in the PowerFLOW ${ }^{\circledR}$ spectrum. The harmonic at $5 \mathrm{kHz}$ is also quite strong. This cavity is covered in this experimental run, so these tones are not seen in the experimental spectra. As in the other cases, the array processing significantly lowers the experimental spectra compared with the single microphone result, but there is little change with the simulation. The agreement between the experiment and simulation is not as good for this configuration as the predicted spectra is 3-5 dB higher over most of the frequency range. The difference between the experimental flap-deployed spectra in Fig. 3 and flap-retracted in 26 is about $3 \mathrm{~dB}$, but the spectra from the simulations does not change significantly for the two configurations. Typically, the deployment of the flap results in lower velocities around the gear, which in turn results in less gear noise over most if not all of the frequency range. However, the flap tips make noise, and the gear wake can interact with the flap when the flap is deployed, which is another potential source of noise. Amongst these competing factors, the experiment indicates that the reduction in noise from the gear is the most significant. Nonetheless, in the PowerFLOW ${ }^{\circledR}$ simulations, the gear itself is not making most of the noise but the gear cavity. If the walls of the cavity are excluded from the FW-H calculation, then the noise drops significantly and more closely matches with the measured values. The experimental cavity does make noise, but primarily at low frequencies and, even then, at lower levels than seen in the simulation. However, the cavity in the experiment was filled with tubes and wires from instrumentation that changed the cavity volume. In addition, the turbulent boundary layer on the wing bottom surface going over the cavity leading edge is not well represented in the simulations. The actual boundary layer in the experiment is much less coherent, and the small features in the cavity further act to break up the large-scale shedding coming off of the upstream cavity lip. The boundary layer state and these small components were not included in the simulations, and their absence appears to be the cause of the discrepancy.

DAMAS beamform maps for a frequency of $3 \mathrm{kHz}$ are presented in Fig. 27. Maps at lower frequencies look very similar to those at this frequency. Although the flap appears deployed in the aircraft images, the flap was set at $0^{\circ}$ in both the experiment and simulation. At $3 \mathrm{kHz}$, the DAMAS maps are similar between the experiment and PowerFLOW ${ }^{\circledR}$ simulation. The source around the gear is about the same size, but some additional although relatively weak random noise is evident in the experimental result. The CLEAN-SC maps (Fig. 28) show much more compact sources than the DAMAS maps, in contrast to what was observed for the other configurations where the DAMAS sources tended to be more compact. Recall that the sum of the squared pressure values from DAMAS image maps produce the total noise generated by a region, so the spot size in the DAMAS maps are probably more representative of the extent of the source, whereas the "resolution" parameter set to 3 inches in the AVEC CLEAN-SC processing is limiting the spot size in the CLEAN-SC results.

At $8 \mathrm{kHz}$, the DAMAS map (Fig. 29) for the experiment exhibits considerable randomness, probably because the noise from the model is weaker for this case, allowing for more contamination from background levels of extraneous noise. The PowerFLOW ${ }^{\circledR}$ result is quite clean and clearly identifies the gear as the source. The CLEAN-SC maps in Fig. 30 also clearly identify the gear, but the location of the source is slightly different between the experiment and simulation. The image maps for a frequency of $20 \mathrm{kHz}$ (Figs. 31 and 32) are similar to those at $8 \mathrm{kHz}$, although the exact location of the primary sources in the CLEAN-SC maps have moved slightly.

\section{Conclusions}

A preliminary assessment of the usefulness of synthetic microphone array processing for a semi-span aircraft model 
has been completed. Despite the relatively short time records available from computational simulations, beamform maps generated from the synthetic array time records are in good agreement with those obtained from the experiment, and the visual appearance of the sources is generally much better with the synthetic data. Clearly, the absence of tunnel background noise and realistic, thick shear layers in the simulations is advantageous for array processing, especially the DAMAS algorithm. Furthermore, the spectrum obtained from DAMAS processing of synthetic array data is virtually identical to that of the center microphone of the array, indicating that the array processing is not excluding any extraneous sources. The sources must be uncorrelated and the propagation between the source and microphone adequately represented by uniform flow. The implication is that array processing of synthetic data for this problem does not improve spectral comparisons with experiment, but the beamform maps do provide an additional means of comparison that can reveal differences that cannot be ascertained from the spectra alone.

The short time records in the simulations are likely to be an issue when the array data is processed in much narrower bands than the $171 \mathrm{~Hz}$ used in the present analysis. However, the choice of $171 \mathrm{~Hz}$ was driven by the requirements of the DAMAS algorithm, and not the record lengths. Nonetheless, $171 \mathrm{~Hz}$ can be too large to isolate some phenomena that may be of interest.

Although the beamform map comparisons between the experiment and two computations are generally in very good agreement, some of the frequencies chosen for presentation were selected to highlight differences. In one case, the maps showed that one calculation had the primary source at the outboard flap edge whereas the other had it at the inboard edge. Just because spectral levels are similar does not mean that the sources are the same, and the beamform maps showed their utility by identifying some discrepancies. Although some plausible explanations for the differences were posited, the real advantage of the simulations is the availability of the near field data that can now be interrogated in an attempt to understand the differences. Although our current coupling of the near- to far-field through the FW-H equation can help localize the sources, it does not directly provide any insight into the physical mechanisms giving rise to the noise. Indeed, the non-radiating, hydrodynamic fluctuations in the near-field are much more intense than acoustic fluctuations, making it difficult to ascertain the coupling between the two. Nonetheless, the FW-H and beamform maps indicate what regions should be interrogated in the volume CFD data, and they indicate the frequencies of interest. Synthetic beamforming could be used to assess the effectiveness of noise reduction strategies in a more quantitative fashion, and to reveal when changes have unintended consequences and create sources in new locations.

The results presented also highlight some differences in the DAMAS and CLEAN-SC array processing techniques. Although both performed well in most cases, both exhibit random sources at higher frequencies. For DAMAS, the synthetic array beamform maps are much cleaner than those obtained using experimental data indicating an increased sensitivity to background noise and other real tunnel effects. CLEAN-SC appears to be less sensitive to extraneous noise sources, but still exhibits a frequency dependence. The current results highlight how synthetic array data from CFD simulations can be useful for analyzing and evaluating array processing algorithms, and this will hopefully lead to improvements in their design.

\section{Acknowledgments}

The authors would like to thank Dr. Raymond Mineck for providing the FUN3D results. This work was supported by the NASA Integrated System Research Program’s Environmentally Responsible Aviation Project.

\section{References}

\footnotetext{
${ }^{1}$ Khorrami, M. R., Humphreys, W. M., Lockard, D. P., and Ravetta, P. A., "Aeroacoustic Evaluation of Flap and Landing Gear Noise Reduction Concepts," AIAA Paper 2014-2478, 2014.

${ }^{2}$ Ffowcs Williams, J. E. and Hawkings, D. L., "Sound Generation by Turbulence and Surfaces in Arbitrary Motion," Philosophical Transactions of the Royal Society, Vol. A264, No. 1151, 1969, pp. 321-342.

${ }^{3}$ Marotta, T. R., Lieber, L. S., and Dougherty, R. P., "Validation of Beamforming Analysis Methodology with Synthesized Acoustic Time History Data: Sub-Scale Fan Rig System,” AIAA Paper 2014-3068, 2014.

${ }^{4}$ Nelson, C. C., Cain, A. B., Raman, G., Chan, T., Saunders, M., Noble, J., Engeln, R., Dougherty, R. P., Brentner, K. S., and Morris, P. J., "Numerical Studies of Wind Turbine Acoustics," AIAA Paper 2012-0006, 2012.

${ }^{5}$ Nelson, C., Cain, A. B., Dougherty, R. P., Brentner, K. S., and Morris, P. J., "Application of Synthetic Array Techniques for Improved Simulations of Hot Supersonic Jet Noise," AIAA Paper 2015-0507, 2015.

${ }^{6}$ Neuhart, D., Hannon, J., and Khorrami, M. R., "Aerodynamic Measurements of a Gulfstream Aircraft Model With and Without Noise Reduction Concepts," AIAA Paper 2014-2477, 2014.

${ }^{7}$ Yao, C.-S., Jenkins, L. N., Bartram, S. M., Jerome, H., Khorrami, M. R., and Mace, W. D., "Flow-Field Investigation of Gear-Flap Interaction on a Gulfstream Aircraft Model," AIAA Paper 2014-2479, 2014.

${ }^{8}$ Khorrami, M. R., Fares, E., and Casalino, D., “Towards Full Aircraft Airframe Noise Prediction: Lattice Boltzmann Simulations,” AIAA Paper 2014-2481, 2014.

${ }^{9}$ Khorrami, M. R. and Mineck, R. E., “Towards Full Aircraft Airframe Noise Prediction: Detached Eddy Simulations,” AIAA Paper 2014-2480, 2014.
} 
${ }^{10}$ AVEC Phased Array Software, Ver 3.11, AVEC, Inc., Blacksburg, VA, URL: http://www.avec-engineering.com/products.html [cited March 17, 2015].

${ }^{11}$ Sijtsma, P., "CLEAN Based on Spatial Source Coherence," AIAA Paper 2007-3436, 2014.

${ }^{12}$ Brooks, T. F. and Humphreys, W. H., "A Deconvolution Approach for the Mapping of Acoustic Sources (DAMAS) Determined from Phased Microphone Arrays," Journal of Sound and Vibration, Vol. 294, 2006, pp. 856-879.

${ }^{13}$ Humphreys, W. M., Brooks, T. F., Bahr, C. J., Spalt, T. B., Bartram, S. M., Culliton, W., and Becker, L., "Development of a Microphone Phased Array Capability for the Langley 14- by 22-foot Subsonic Tunnel," AIAA Paper 2014-2343, 2014.

${ }^{14}$ AIAA Aeroacoustics Technical Committee Discussion Group on Array Analysis Methods for Aeroacoustics, URL: https://info.aiaa.org/tac/ASG/AATC/Lists/Array Analysis Methods for Aeroacoustics/AllItems.aspx [cited April 7, 2015].

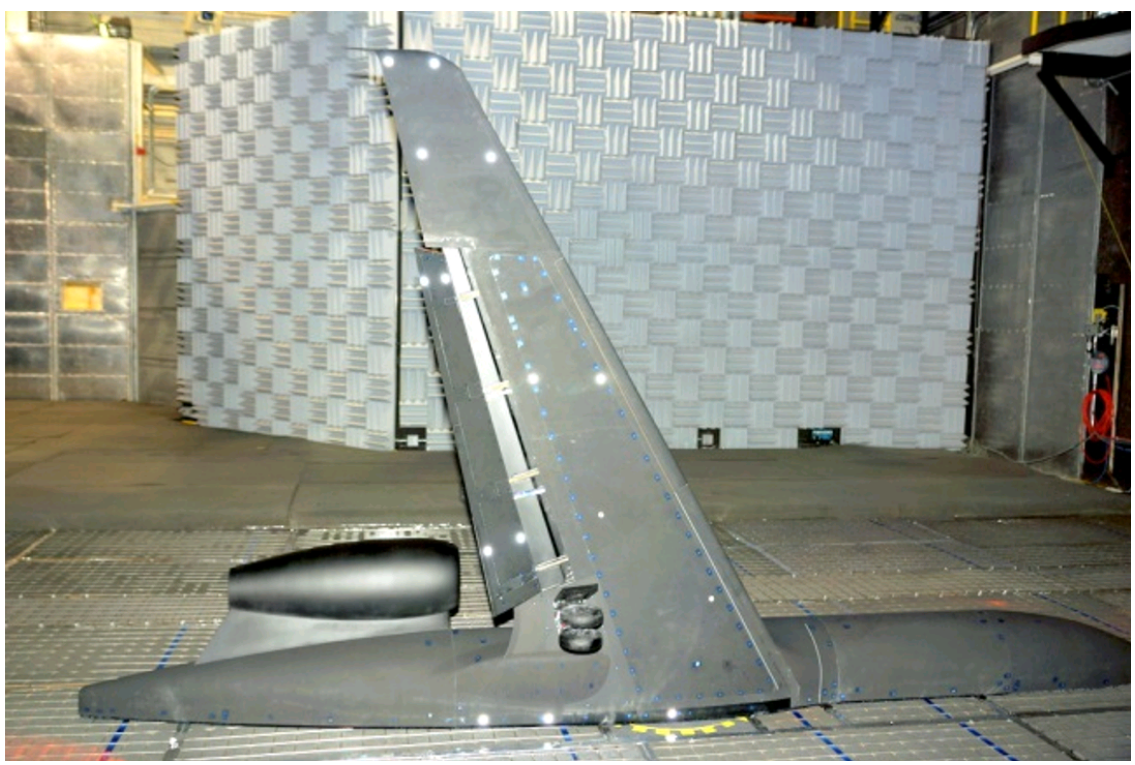

Figure 1. View of Model from Array

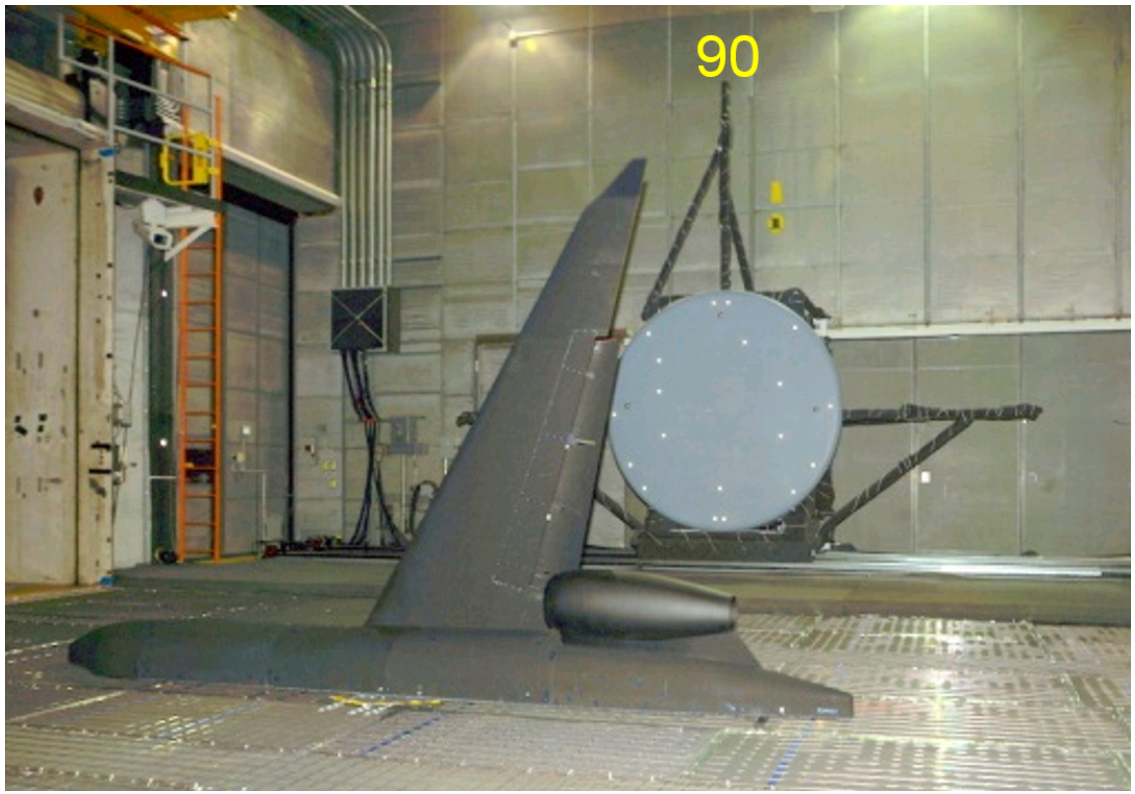

Figure 2. View of Array in the $90^{\circ}$ Flyover Position 


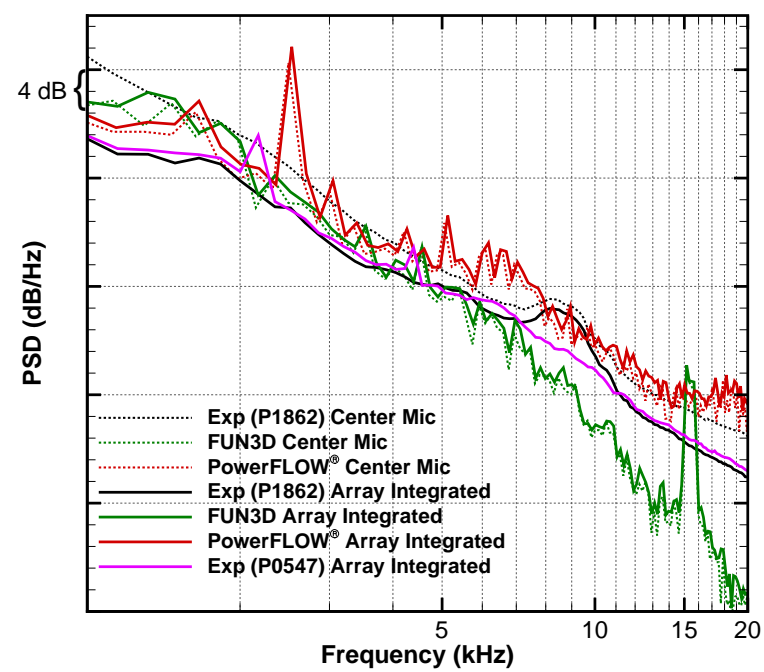

(a) DAMAS

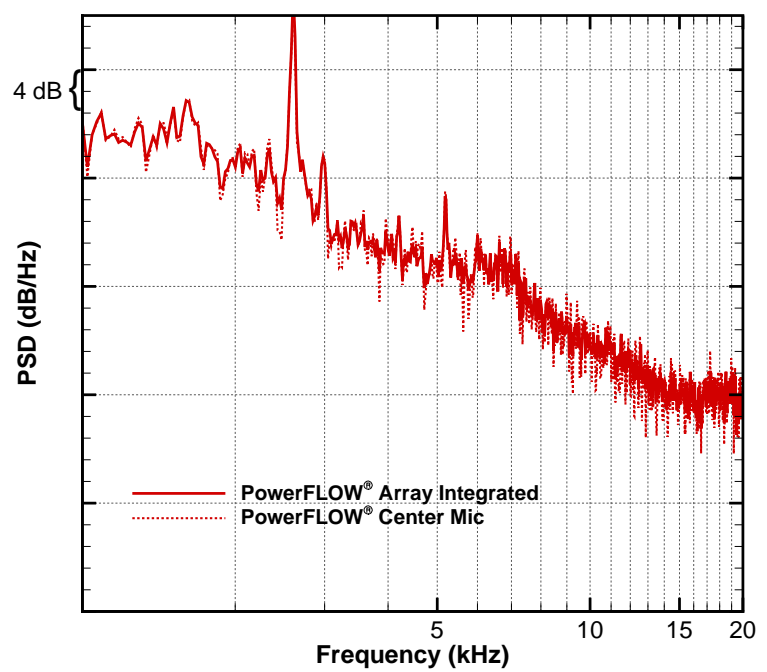

(b) Exa CLEAN-SC

Figure 3. Comparison of Integrated Array Beamform and Center Microphone Spectra. Gear on, Flaps at $39^{\circ}$.

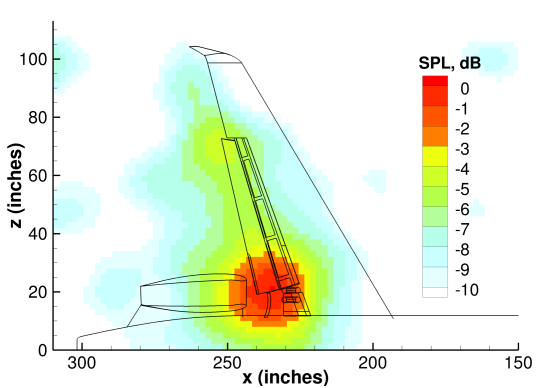

(a) Experimental

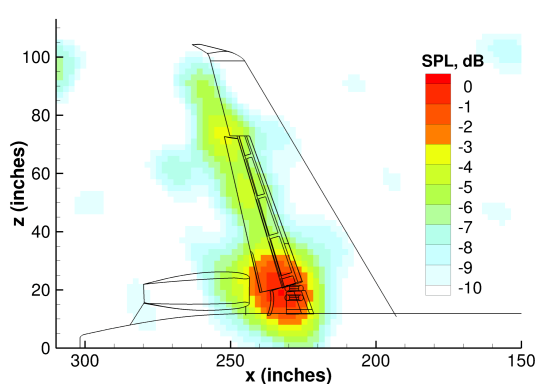

(b) PowerFLOW ${ }^{\circledR}$ Simulated

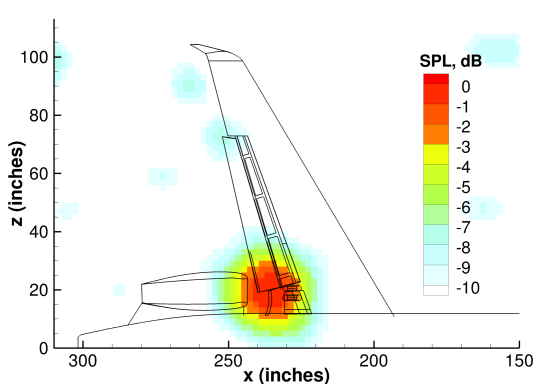

(c) FUN3D Simulated

Figure 4. Conventional Beamforming Results from UDAMAS Code for $f=2.80 \mathrm{kHz}$, Gear on, Flaps at $39^{\circ}$.

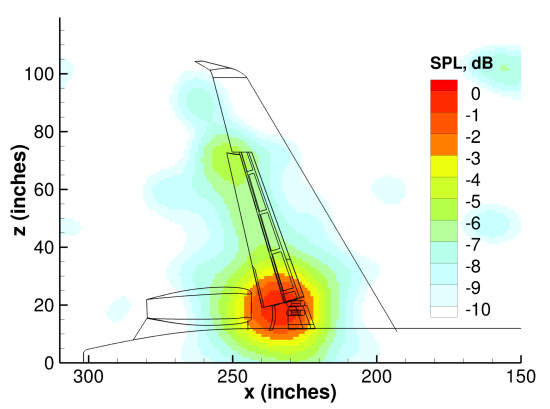

(a) Experimental

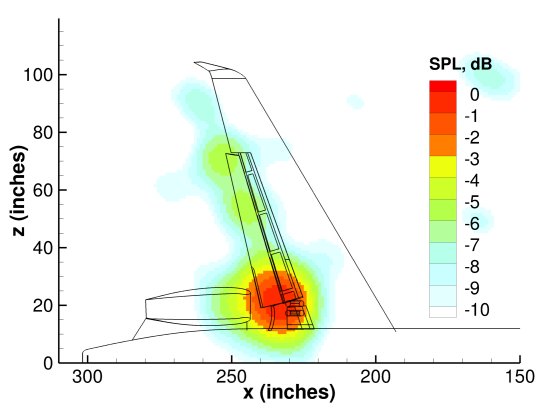

(b) PowerFLOW ${ }^{\circledR}$ Simulated

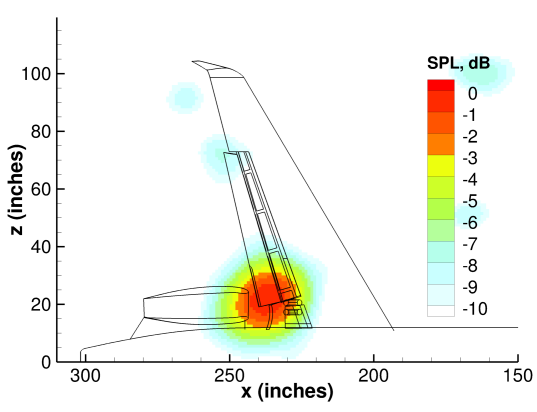

(c) FUN3D Simulated

Figure 5. Conventional Beamforming Results from AVEC Code for $f=2.80 \mathrm{kHz}$, Gear on, Flaps at $39^{\circ}$. 


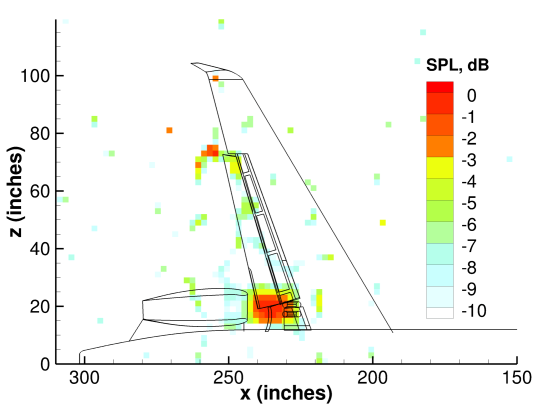

(a) Experimental

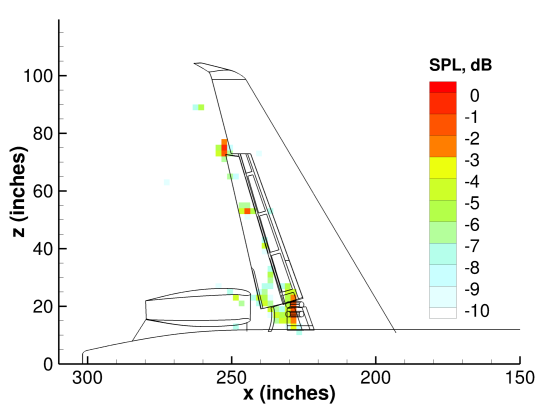

(b) PowerFLOW ${ }^{\circledR}$ Simulated

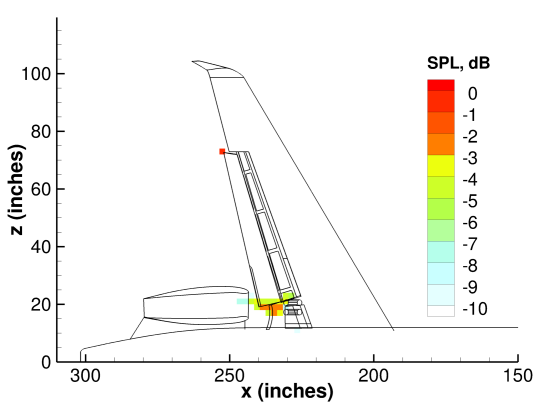

(c) FUN3D Simulated

Figure 6. DAMAS Beamforming Results for $f=2.80 \mathrm{kHz}$, Gear on, Flaps at $39^{\circ}$.

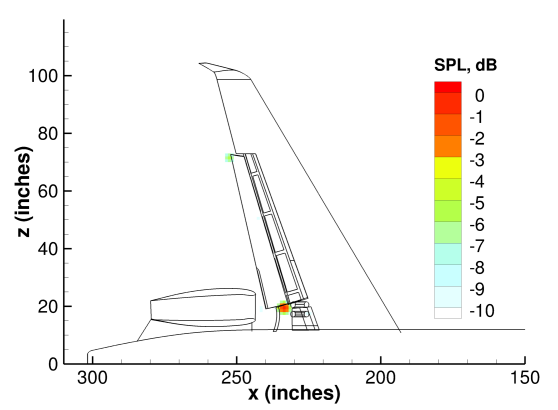

(a) Experimental

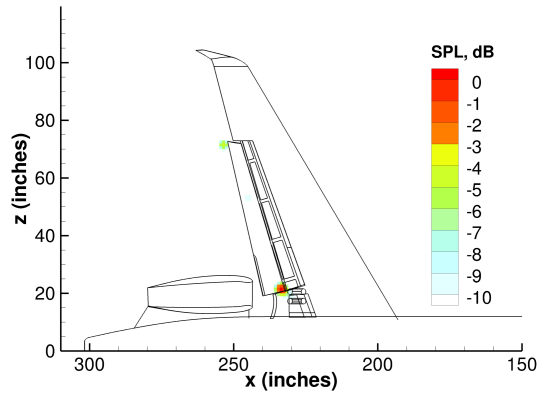

(b) PowerFLOW ${ }^{\circledR}$ Simulated

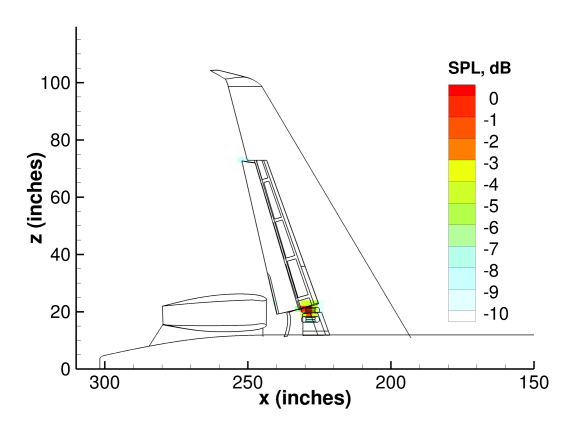

(d) PowerFLOW ${ }^{\circledR}$, Exa Beamformer

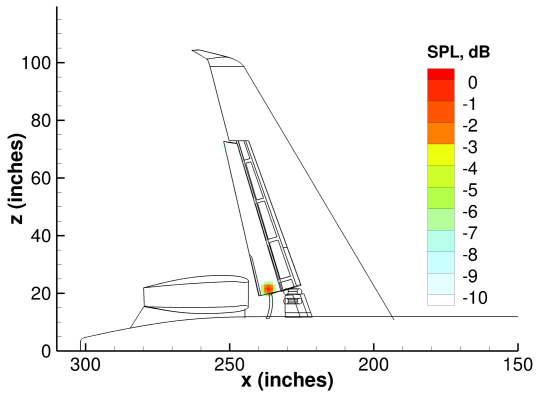

(c) FUN3D Simulated

Figure 7. CLEAN-SC Beamforming Results for $f=2.80 \mathrm{kHz}$, Gear on, Flaps at $39^{\circ}$. AVEC code used unless otherwise noted.

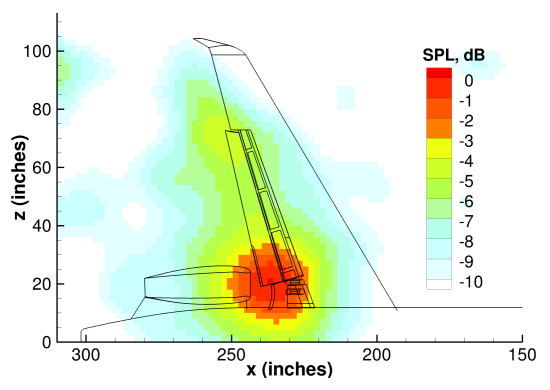

(a) Experimental

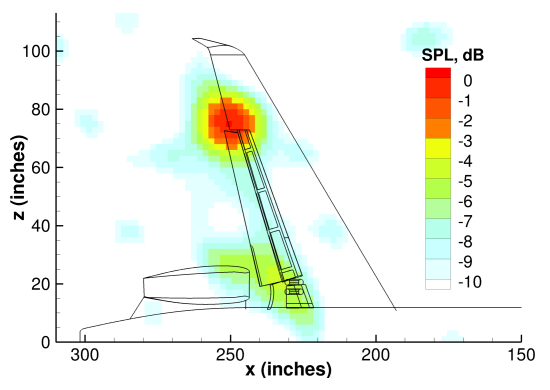

(b) PowerFLOW ${ }^{\circledR}$ Simulated

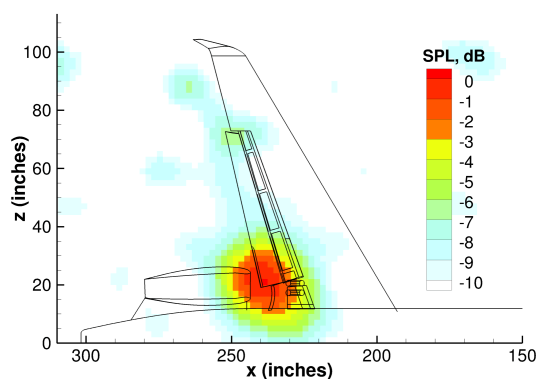

(c) FUN3D Simulated

Figure 8. Conventional Beamforming Results from UDAMAS Code for $f=3.0 \mathrm{kHz}$, Gear on, Flaps at $3^{\circ}$. 


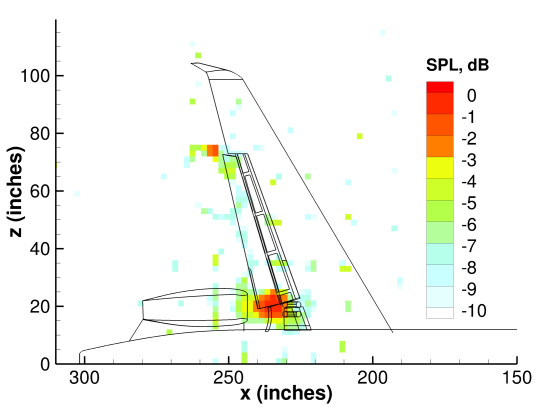

(a) Experimental

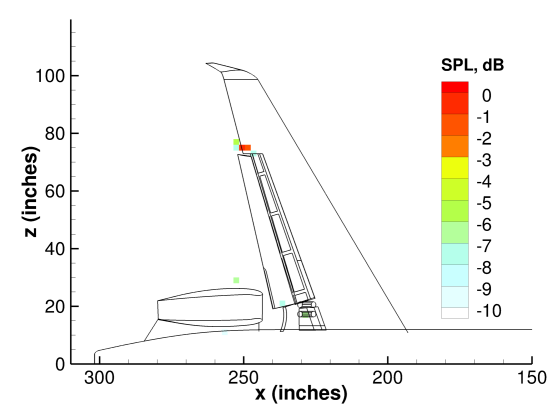

(b) PowerFLOW ${ }^{\circledR}$ Simulated

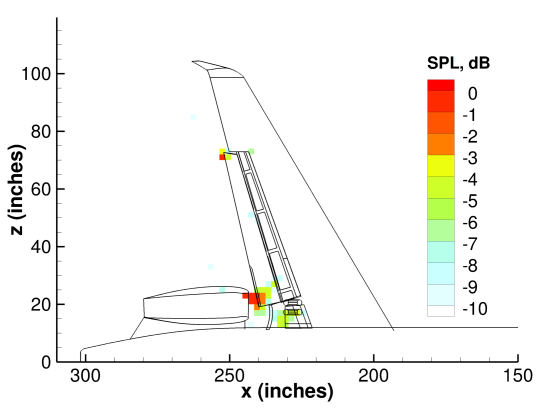

(c) FUN3D Simulated

Figure 9. DAMAS Beamforming Results for $f=3.0 \mathrm{kHz}$, Gear on, Flaps at $39^{\circ}$.

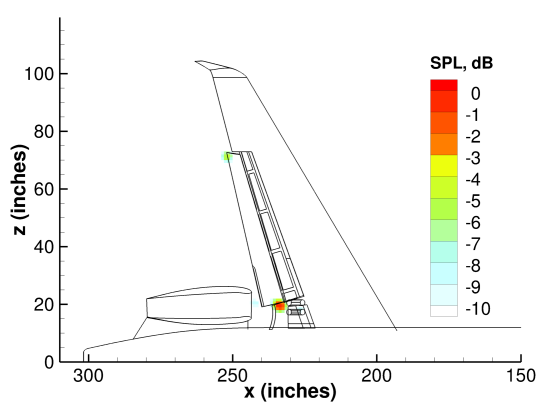

(a) Experimental

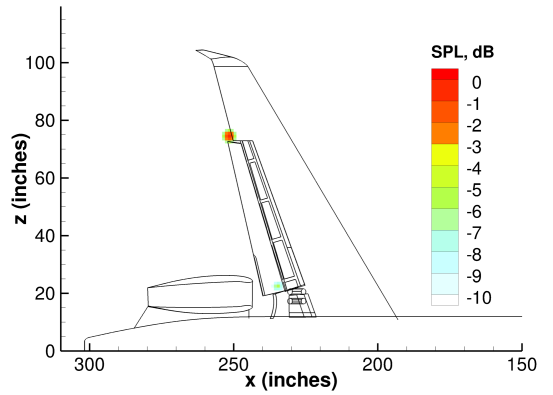

(b) PowerFLOW ${ }^{\circledR}$ Simulated

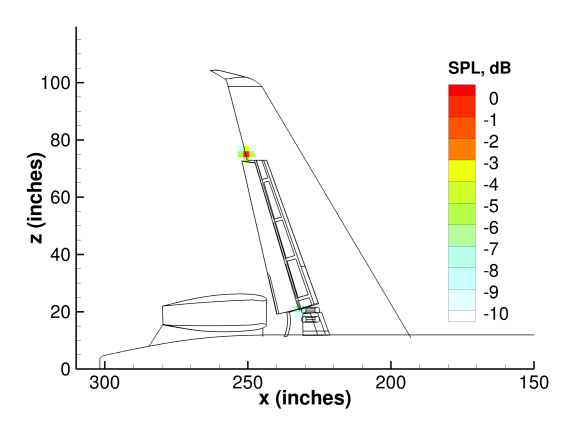

(d) PowerFLOW ${ }^{\circledR}$, Exa Beamformer

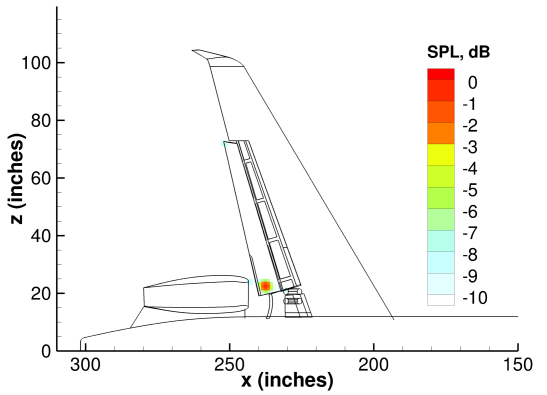

(c) FUN3D Simulated

Figure 10. CLEAN-SC Beamforming Results for $f=3.0 \mathrm{kHz}$, Gear on, Flaps at $39^{\circ}$. AVEC code used unless otherwise noted.

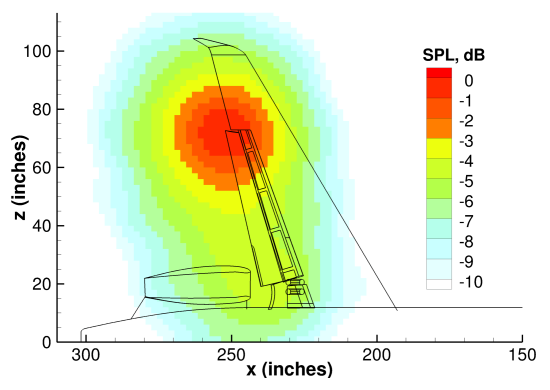

(a) Experimental

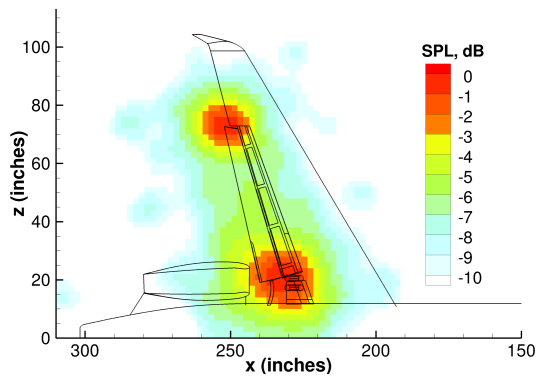

(b) PowerFLOW ${ }^{\circledR}$ Simulated

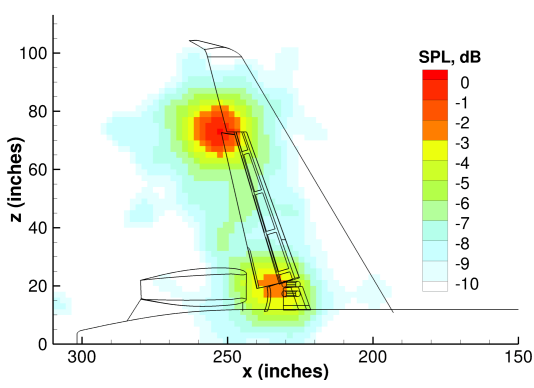

(c) FUN3D Simulated

Figure 11. Conventional Beamforming Results for $f=8.0 \mathrm{kHz}$, Gear on, Flaps at $39^{\circ}$. 


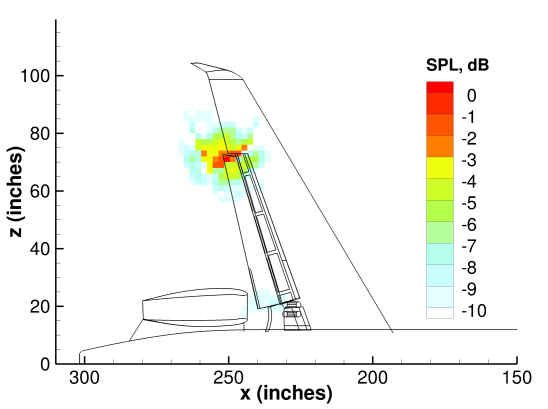

(a) Experimental

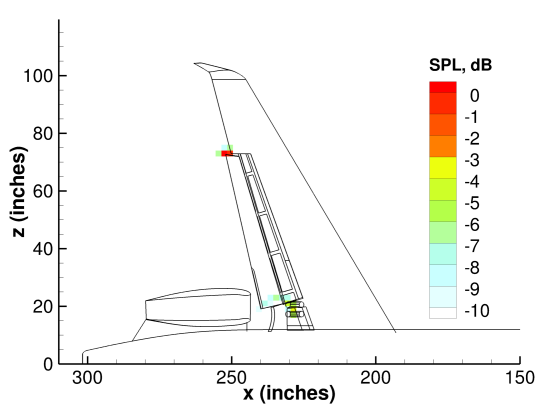

(b) PowerFLOW ${ }^{\circledR}$ Simulated

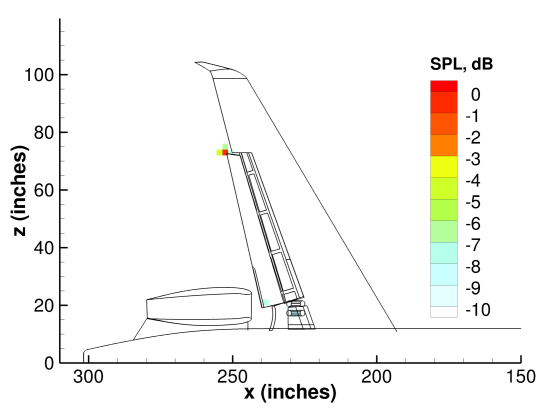

(c) FUN3D Simulated

Figure 12. DAMAS Beamforming Results for $f=8.0 \mathrm{kHz}$, Gear on, Flaps at $39^{\circ}$.

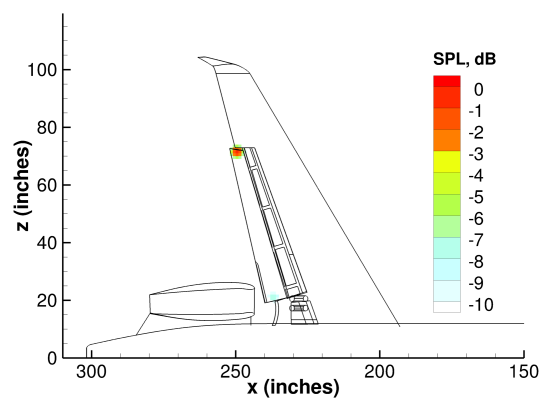

(a) Experimental

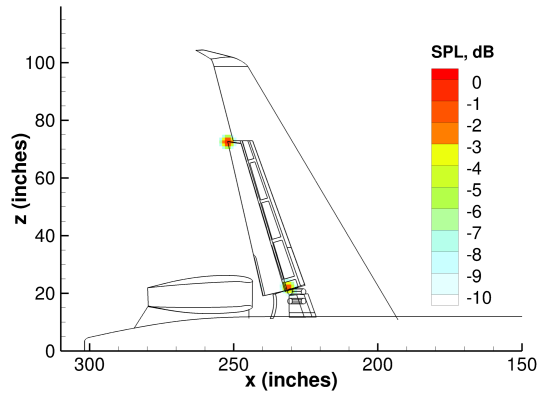

(b) PowerFLOW ${ }^{\circledR}$ Simulated

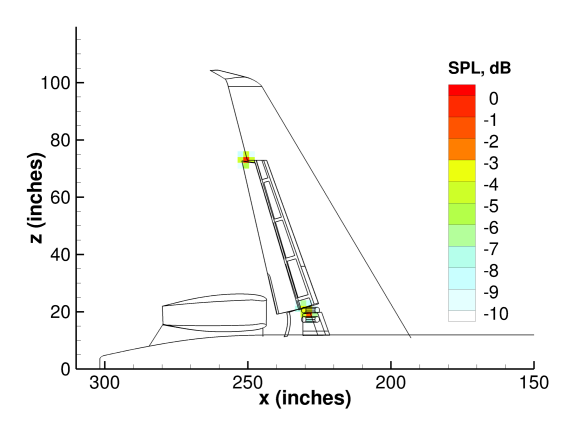

(d) PowerFLOW ${ }^{\circledR}$, Exa Beamformer

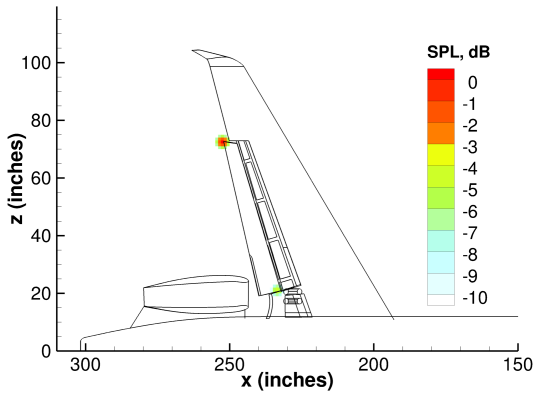

(c) FUN3D Simulated

Figure 13. CLEAN-SC Beamforming Results for $f=8.0 \mathrm{kHz}$, Gear on, Flaps at $3^{\circ}$. AVEC code used unless otherwise noted.

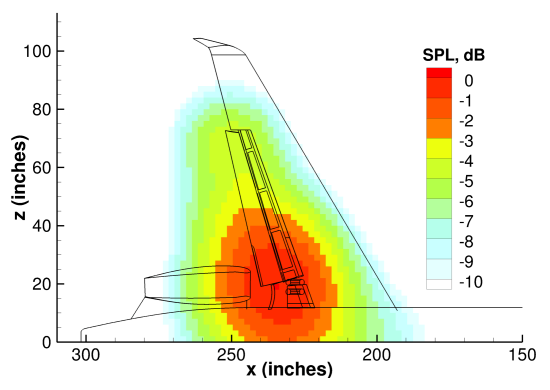

(a) Experimental

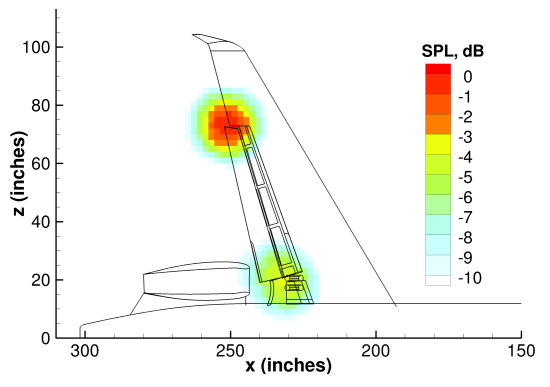

(b) PowerFLOW ${ }^{\circledR}$ Simulated

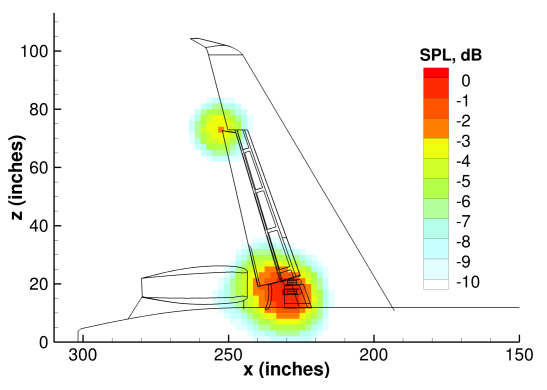

(c) FUN3D Simulated

Figure 14. Conventional Beamforming Results for $f=20.0 \mathrm{kHz}$, Gear on, Flaps at $39^{\circ}$. 


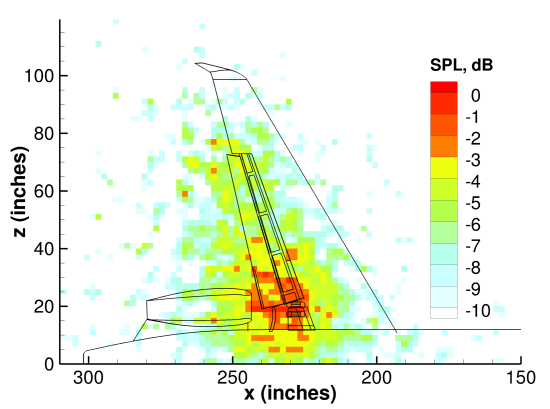

(a) Experimental

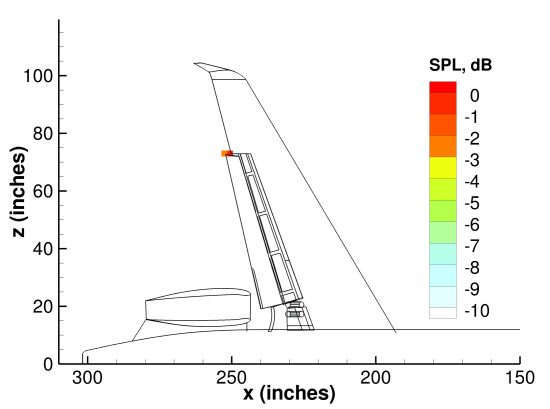

(b) PowerFLOW ${ }^{\circledR}$ Simulated

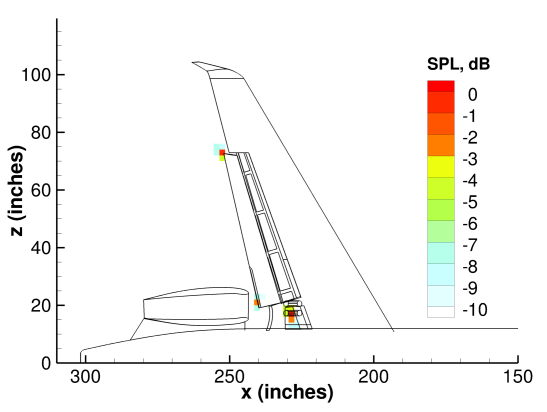

(c) FUN3D Simulated

Figure 15. DAMAS Beamforming Results for $f=20.0 \mathrm{kHz}$, Gear on, Flaps at $39^{\circ}$.

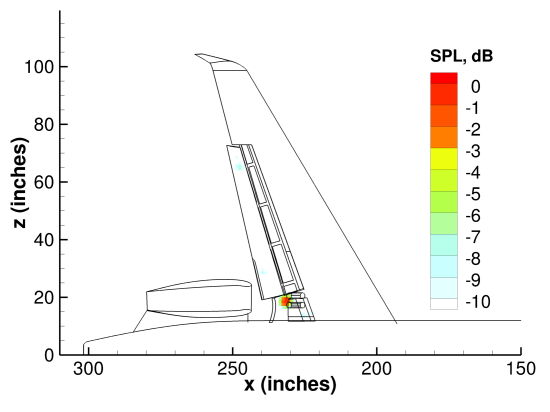

(a) Experimental

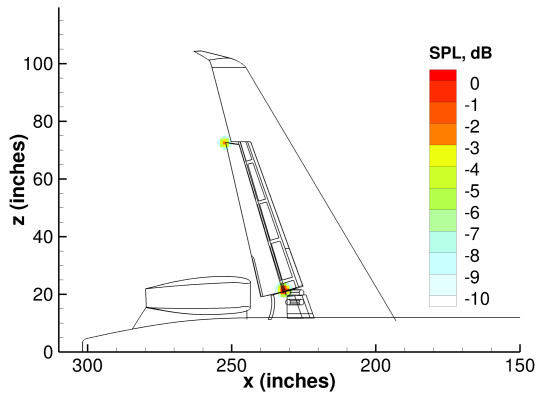

(b) PowerFLOW ${ }^{\circledR}$ Simulated

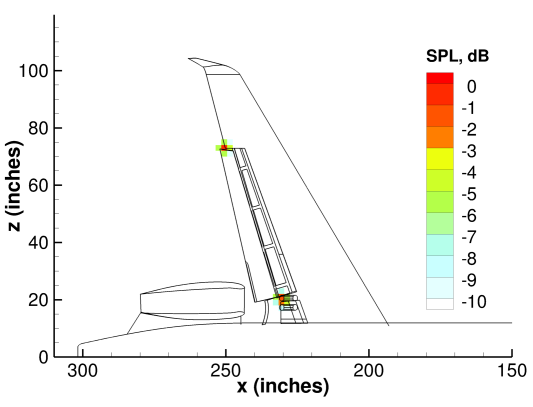

(d) PowerFLOW ${ }^{\circledR}$, Exa Beamformer

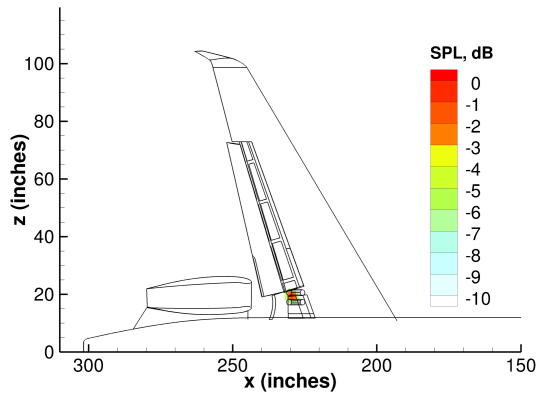

(c) FUN3D Simulated 


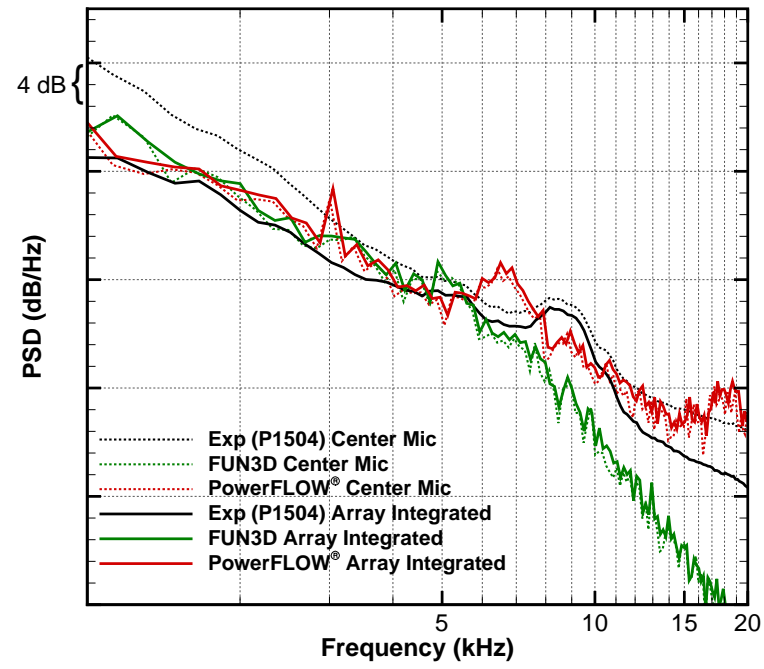

(a) DAMAS

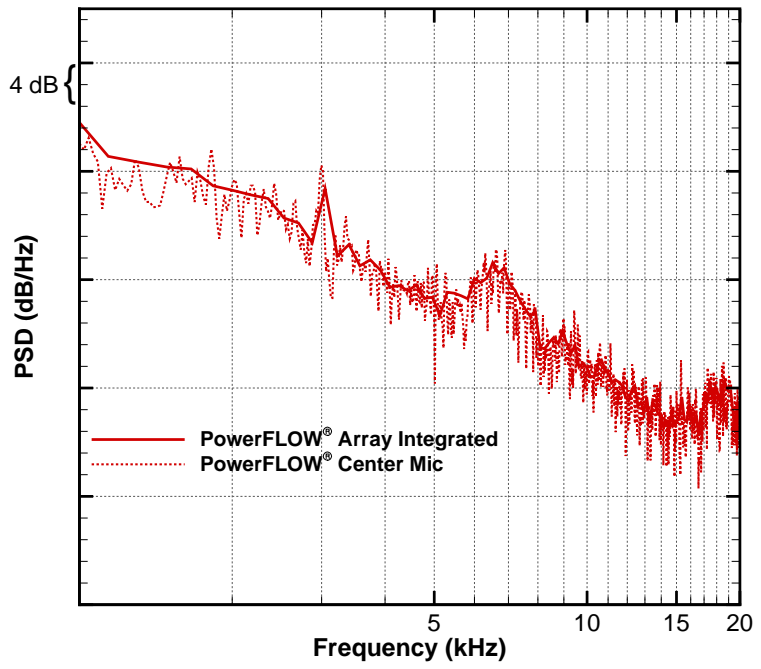

(b) Exa CLEAN-SC

Figure 17. Comparison of Integrated Array Beamform and Center Microphone Spectra. Gear off, Flaps at $39^{\circ}$.

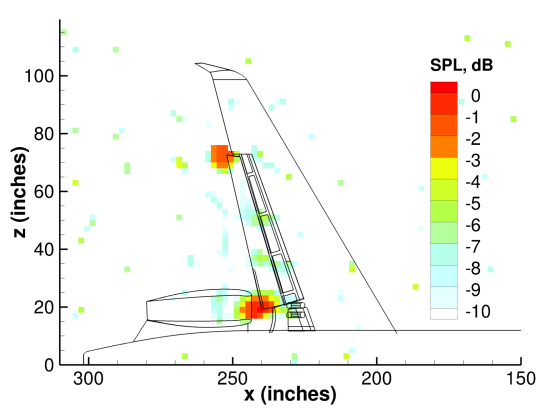

(a) Experimental

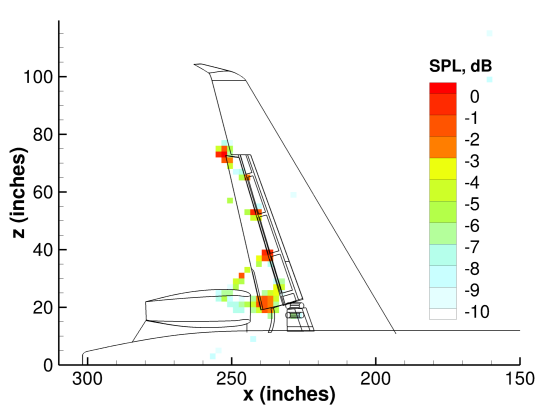

(b) PowerFLOW ${ }^{\circledR}$ Simulated

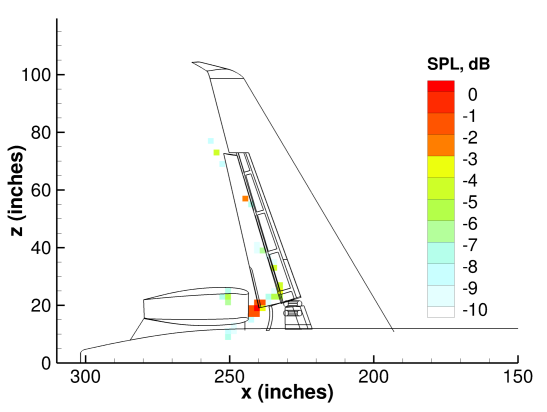

(c) FUN3D Simulated

Figure 18. DAMAS Beamforming Results for $f=2.65 \mathrm{kHz}$, Gear on, Flaps at $3^{\circ}$. 


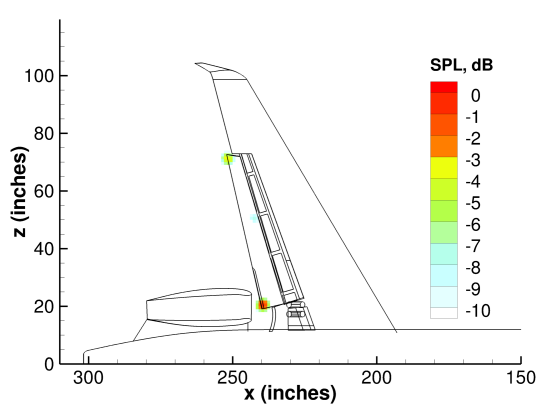

(a) Experimental

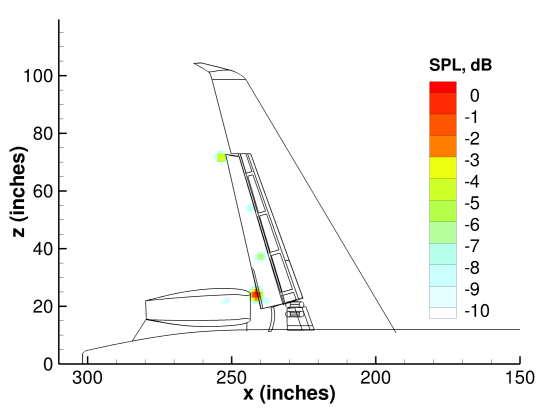

(b) PowerFLOW ${ }^{\circledR}$ Simulated

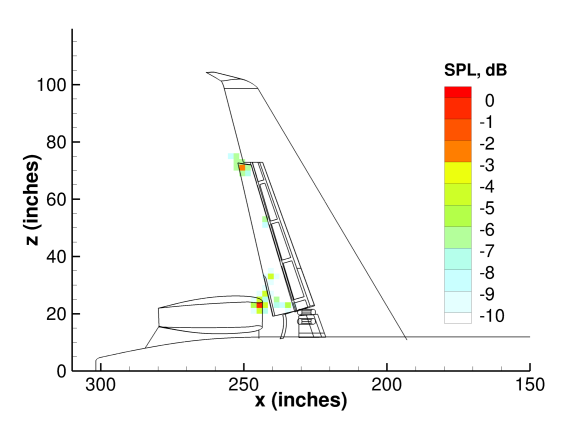

(d) PowerFLOW ${ }^{\circledR}$, Exa Beamformer

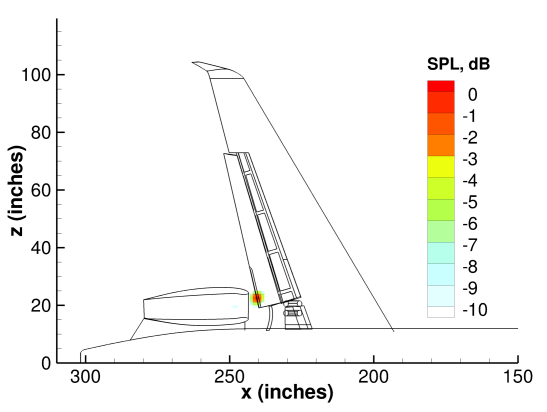

(c) FUN3D Simulated

Figure 19. CLEAN-SC Beamforming Results for $f=2.65 \mathrm{kHz}$, Gear off, Flaps at $39^{\circ}$. AVEC code used unless otherwise noted.

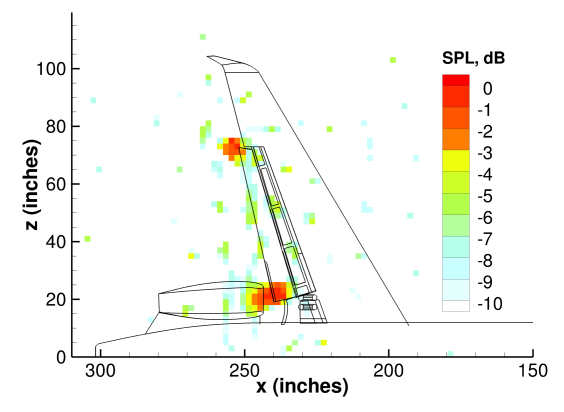

(a) Experimental

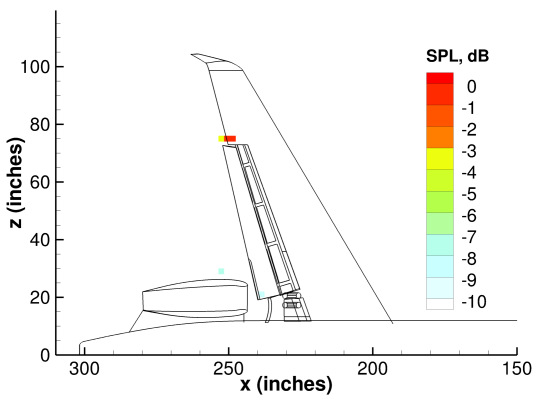

(b) PowerFLOW ${ }^{\circledR}$ Simulated

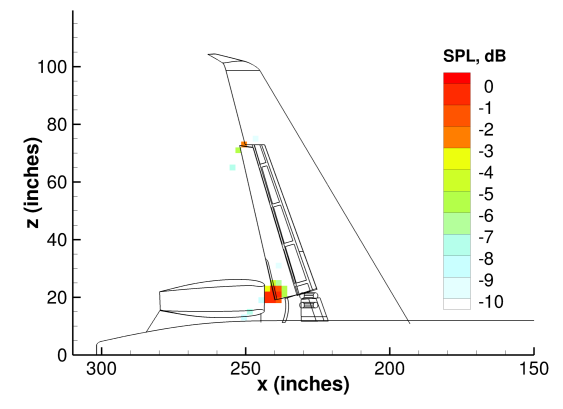

(c) FUN3D Simulated

Figure 20. DAMAS Beamforming Results for $f=3.0 \mathrm{kHz}$, Gear off, Flaps at $39^{\circ}$. 


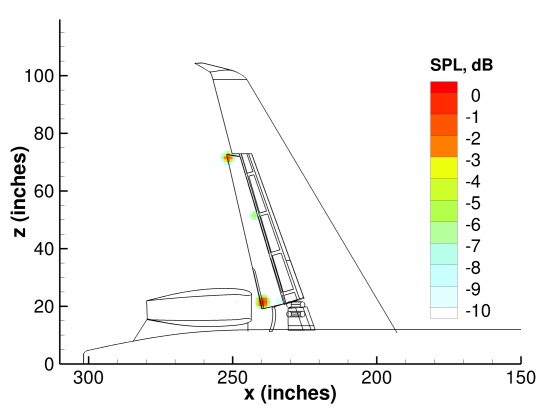

(a) Experimental

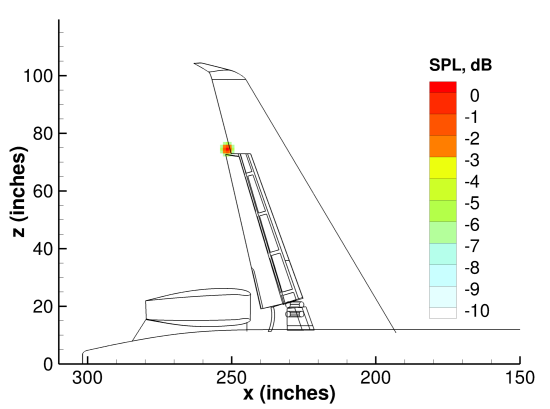

(b) PowerFLOW ${ }^{\circledR}$ Simulated

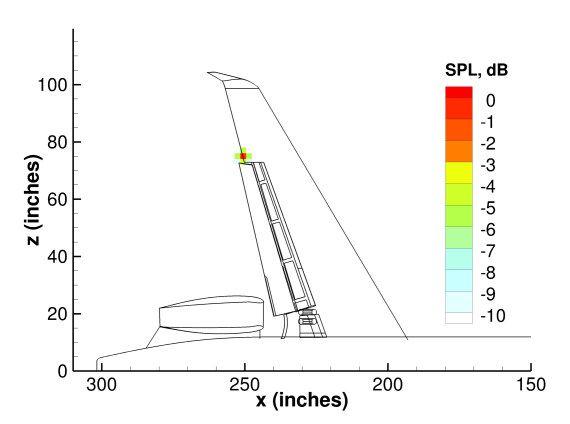

(d) PowerFLOW ${ }^{\circledR}$, Exa Beamformer

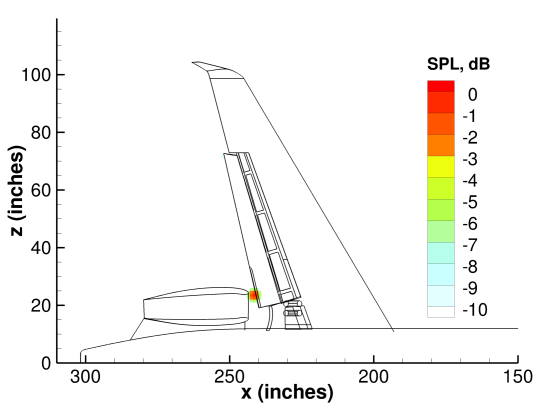

(c) FUN3D Simulated

Figure 21. CLEAN-SC Beamforming Results for $f=3.0 \mathrm{kHz}$, Gear off, Flaps at $39^{\circ}$. AVEC code used unless otherwise noted.

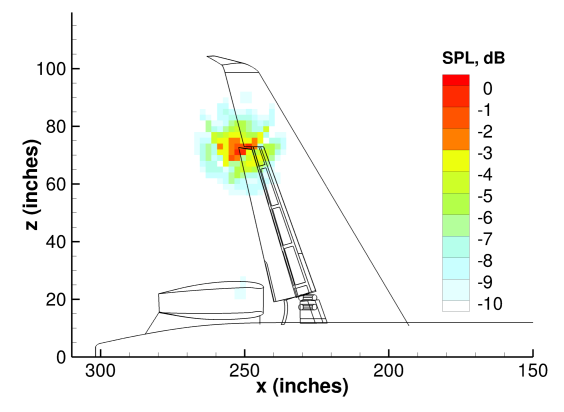

(a) Experimental

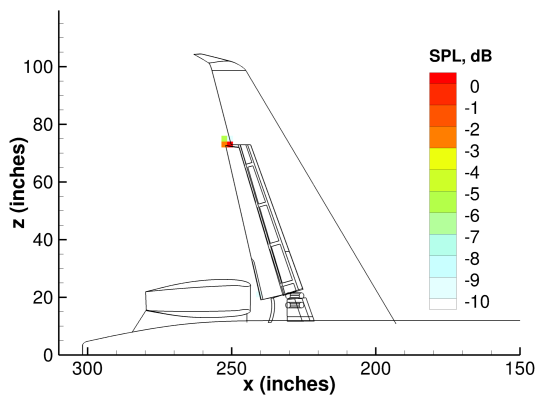

(b) PowerFLOW ${ }^{\circledR}$ Simulated

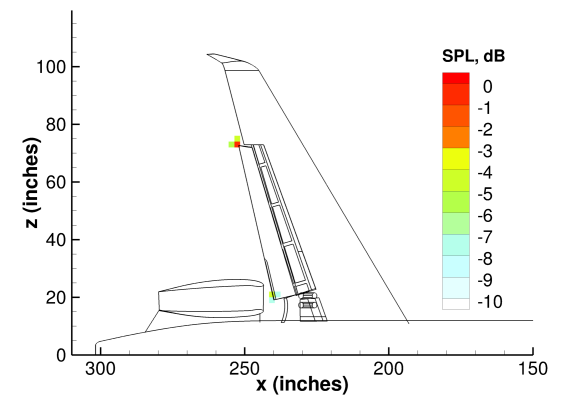

(c) FUN3D Simulated

Figure 22. DAMAS Beamforming Results for $f=8.0 \mathrm{kHz}$, Gear off, Flaps at $39^{\circ}$. 


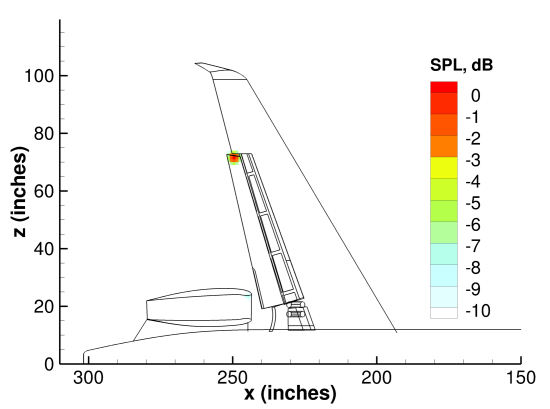

(a) Experimental

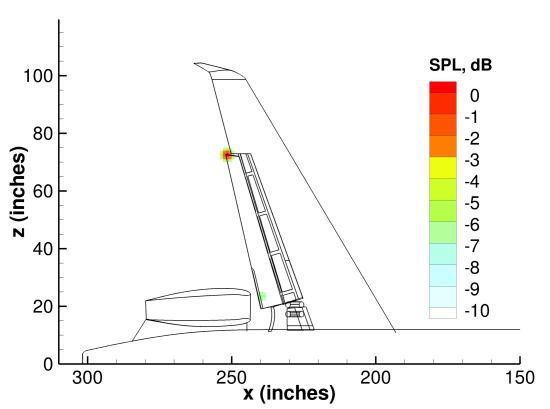

(b) PowerFLOW ${ }^{\circledR}$ Simulated

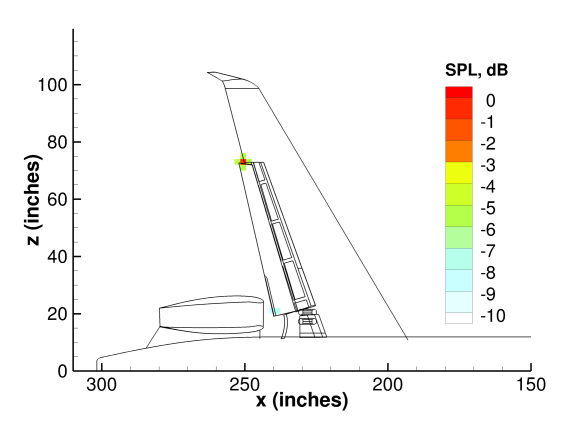

(d) PowerFLOW ${ }^{\circledR}$, Exa Beamformer

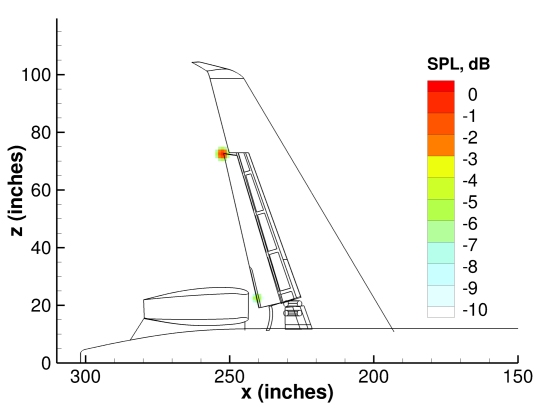

(c) FUN3D Simulated

Figure 23. CLEAN-SC Beamforming Results for $f=8.0 \mathrm{kHz}$, Gear off, Flaps at $39^{\circ}$. AVEC code used unless otherwise noted.

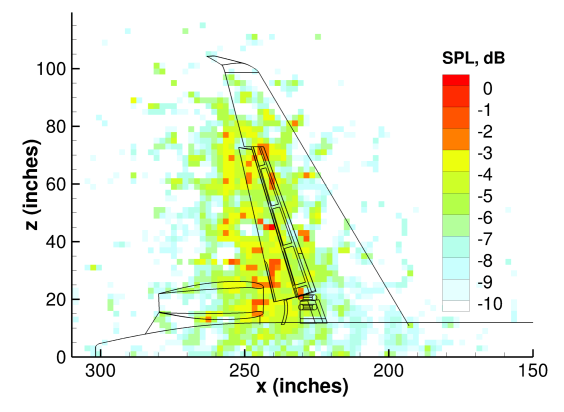

(a) Experimental

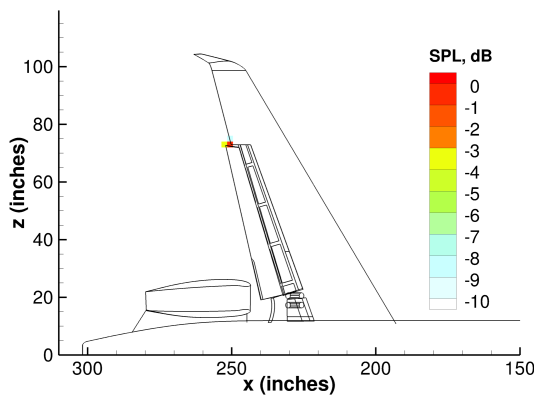

(b) PowerFLOW ${ }^{\circledR}$ Simulated

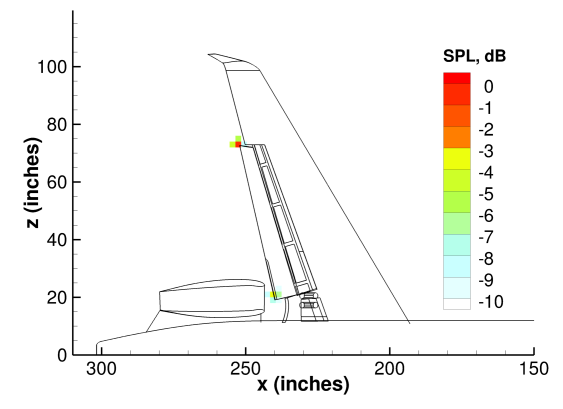

(c) FUN3D Simulated

Figure 24. DAMAS Beamforming Results for $f=20.0 \mathrm{kHz}$, Gear off, Flaps at $3^{\circ}$. 


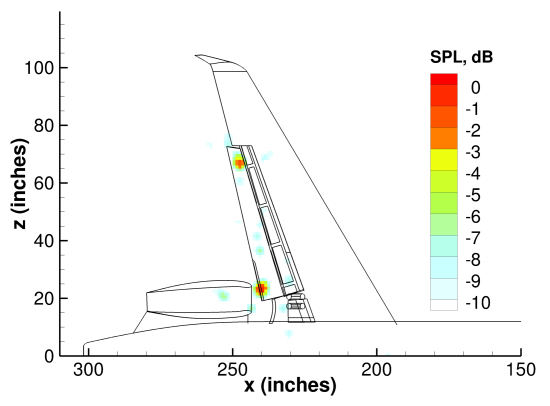

(a) Experimental

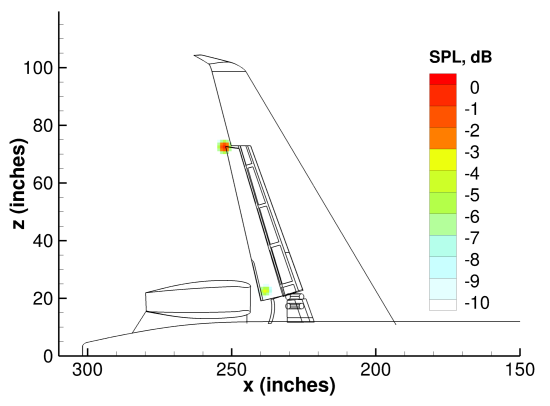

(b) PowerFLOW ${ }^{\circledR}$ Simulated

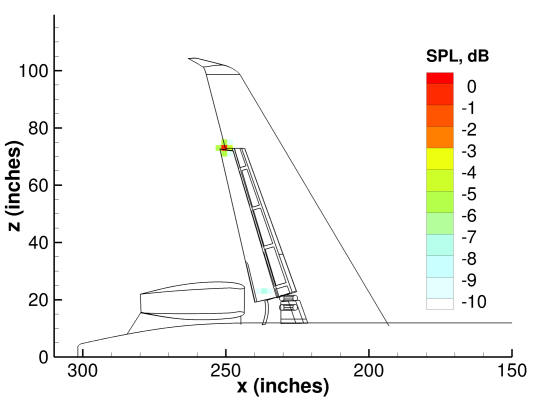

(d) PowerFLOW ${ }^{\circledR}$, Exa Beamformer

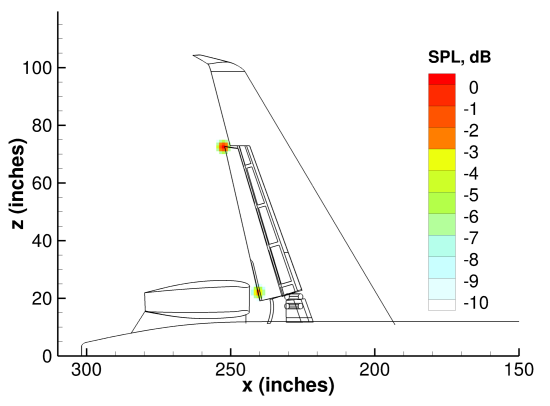

(c) FUN3D Simulated

Figure 25. CLEAN-SC Beamforming Results for $f=20.0 \mathrm{kHz}$, Gear off, Flaps at $39^{\circ}$. AVEC code used unless otherwise noted.

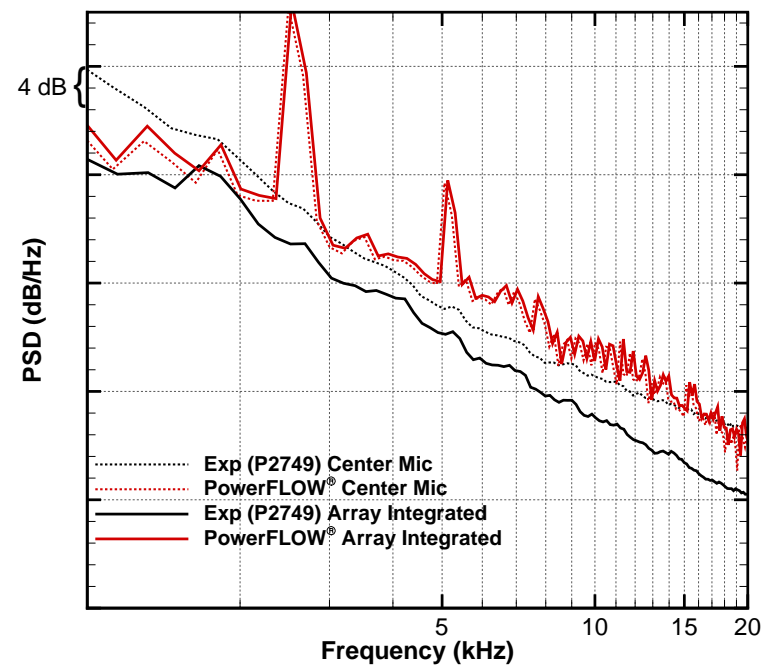

(a) DAMAS

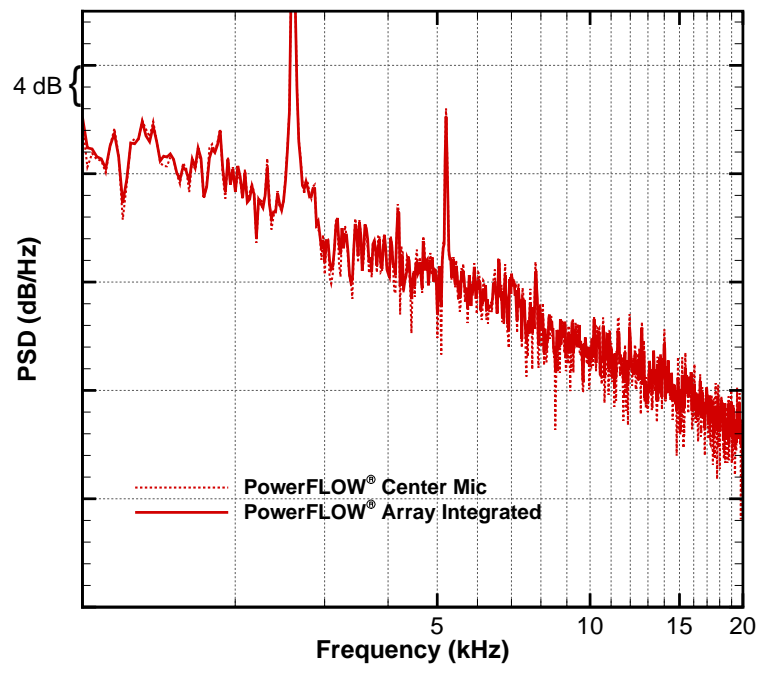

(b) Exa CLEAN-SC

Figure 26. Comparison of Integrated Array Beamform and Center Microphone Spectra. Gear on, Flaps at $39^{\circ}$. 


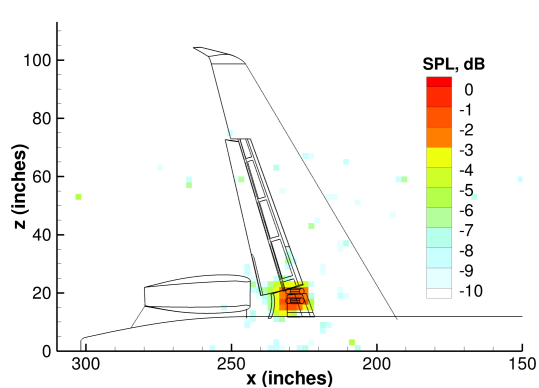

(a) Experimental

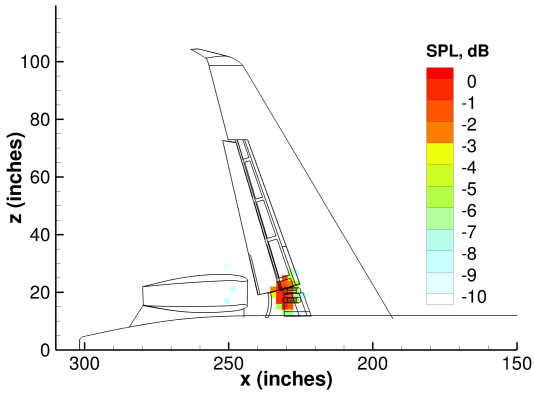

(b) PowerFLOW ${ }^{\circledR}$ Simulated

Figure 27. DAMAS Beamforming Results for $f=3.0 \mathrm{kHz}$, Gear on, Flaps at $0^{\circ}$.

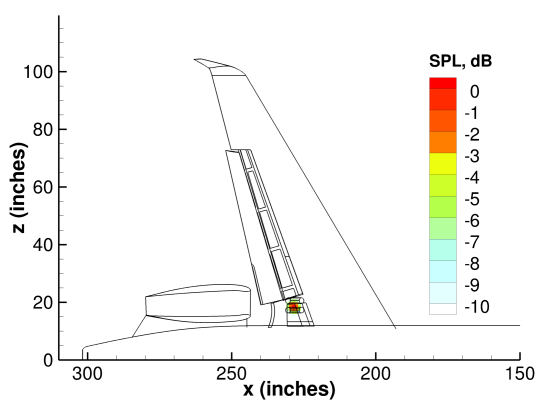

(a) Experimental

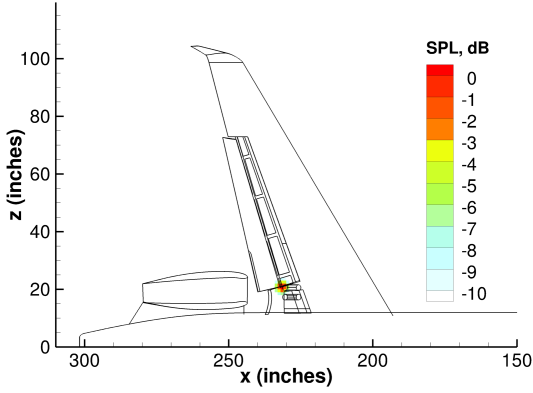

(b) PowerFLOW ${ }^{\circledR}$ Simulated

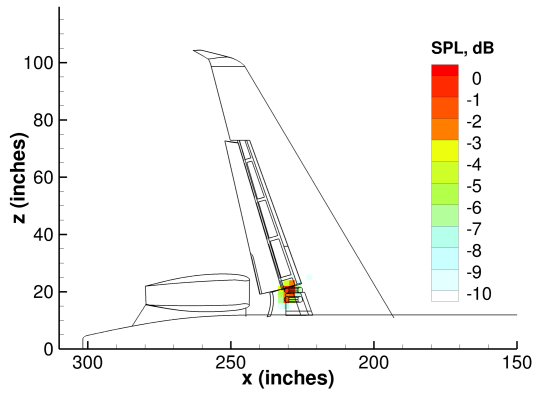

(c) PowerFLOW ${ }^{\circledR}$, Exa Beamformer

Figure 28. CLEAN-SC Beamforming Results for $f=3.0 \mathrm{kHz}$, Gear on, Flaps at $0^{\circ}$. AVEC code used unless otherwise noted.

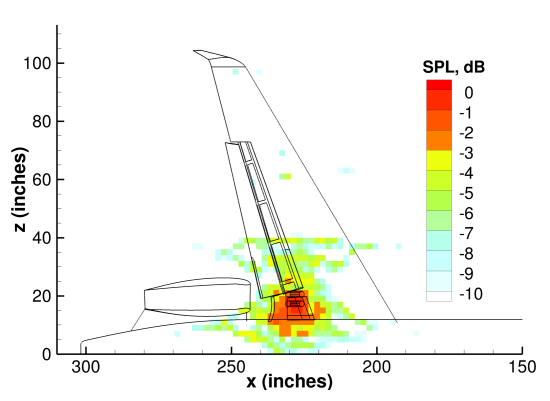

(a) Experimental

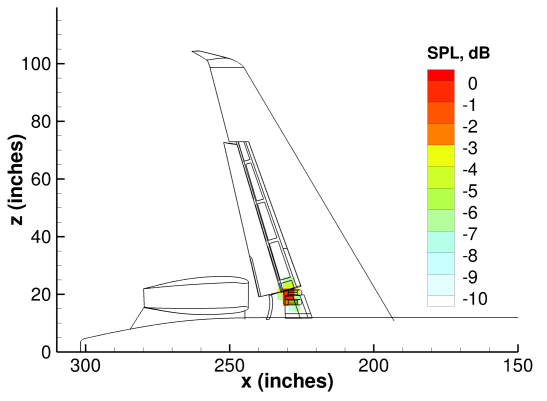

(b) PowerFLOW ${ }^{\circledR}$ Simulated

Figure 29. DAMAS Beamforming Results for $f=8.0 \mathrm{kHz}$, Gear on, Flaps at $0^{\circ}$.

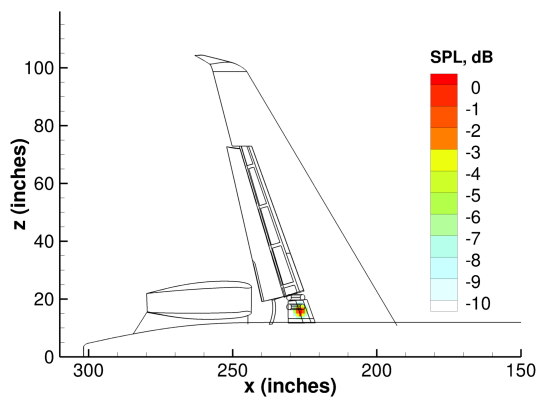

(a) Experimental

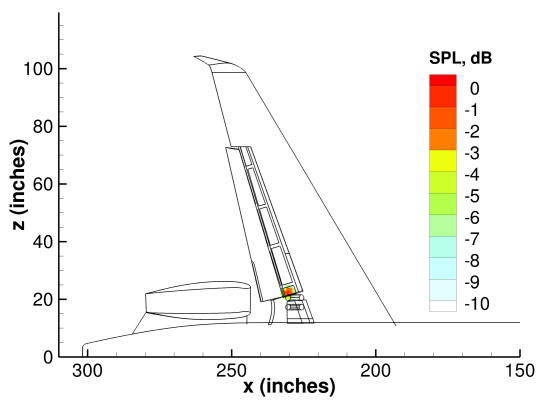

(b) PowerFLOW ${ }^{\circledR}$ Simulated

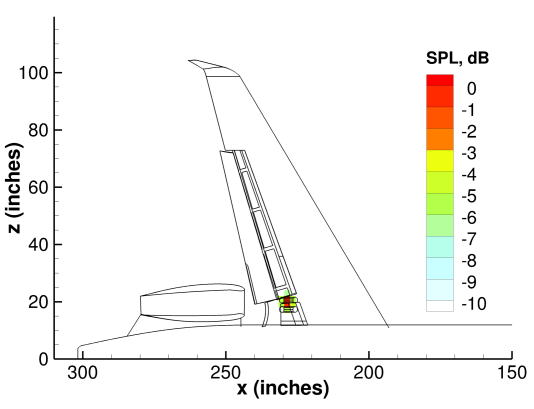

(c) PowerFLOW ${ }^{\circledR}$, Exa Beamformer

Figure 30. CLEAN-SC Beamforming Results for $f=8.0 \mathrm{kHz}$, Gear on, Flaps at $0^{\circ}$. AVEC code used unless otherwise noted. 


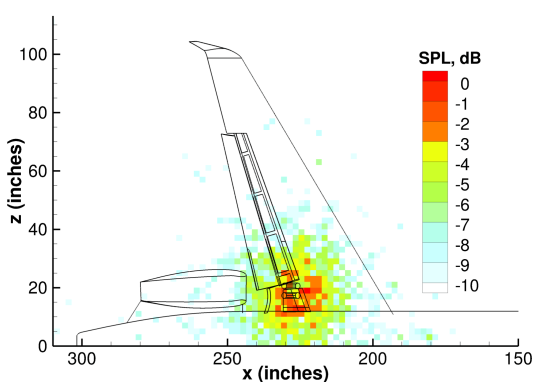

(a) Experimental

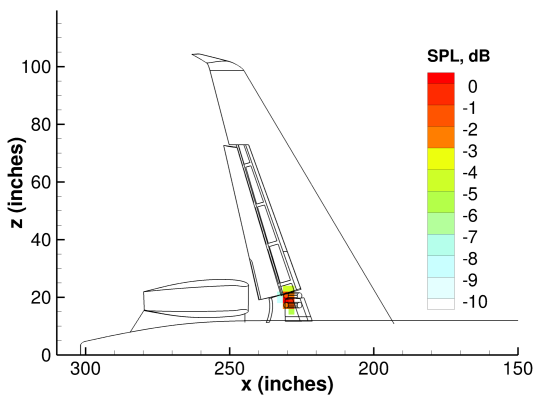

(b) PowerFLOW ${ }^{\circledR}$ Simulated

Figure 31. DAMAS Beamforming Results for $f=20.0 \mathrm{kHz}$, Gear on, Flaps at $0^{\circ}$.

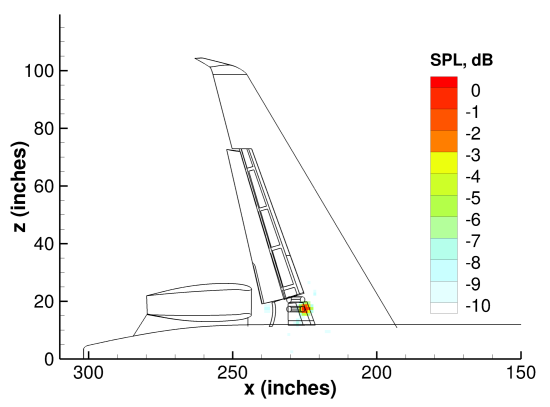

(a) Experimental

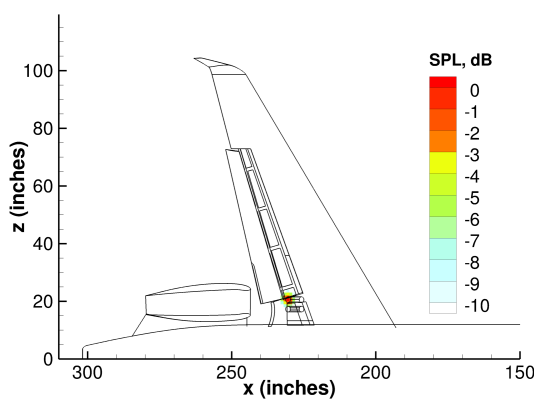

(b) PowerFLOW ${ }^{\circledR}$ Simulated

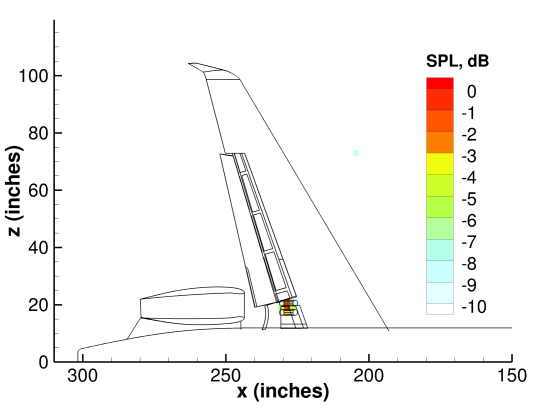

(c) PowerFLOW ${ }^{\circledR}$, Exa Beamformer

Figure 32. CLEAN-SC Beamforming Results for $f=20.0 \mathrm{kHz}$, Gear on, Flaps at $0^{\circ}$. AVEC code used unless otherwise noted. 